H. Lang, J. Linn, M. Arnold

Multibody dynamics simulation of geometrically exact Cosserat rods 
(C) Fraunhofer-Institut für Techno- und Wirtschaftsmathematik ITWM 2009

ISSN 1434-9973

Bericht 159 (2009)

Alle Rechte vorbehalten. Ohne ausdrückliche schriftliche Genehmigung des Herausgebers ist es nicht gestattet, das Buch oder Teile daraus in irgendeiner Form durch Fotokopie, Mikrofilm oder andere Verfahren zu reproduzieren oder in eine für Maschinen, insbesondere Datenverarbeitungsanlagen, verwendbare Sprache zu übertragen. Dasselbe gilt für das Recht der öffentlichen Wiedergabe.

Warennamen werden ohne Gewährleistung der freien Verwendbarkeit benutzt.

Die Veröffentlichungen in der Berichtsreihe des Fraunhofer ITWM können bezogen werden über:

Fraunhofer-Institut für Techno- und Wirtschaftsmathematik ITWM Fraunhofer-Platz 1

67663 Kaiserslautern

Germany

Telefon: $\quad 0631 / 31600-0$

Telefax: 0631/31600-1099

E-Mail: info@itwm.fraunhofer.de

Internet: www.itwm.fraunhofer.de 
Das Tätigkeitsfeld des Fraunhofer-Instituts für Techno- und Wirtschaftsmathematik ITWM umfasst anwendungsnahe Grundlagenforschung, angewandte Forschung sowie Beratung und kundenspezifische Lösungen auf allen Gebieten, die für Techno- und Wirtschaftsmathematik bedeutsam sind.

In der Reihe »Berichte des Fraunhofer ITWM « soll die Arbeit des Instituts kontinuierlich einer interessierten Öffentlichkeit in Industrie, Wirtschaft und Wissenschaft vorgestellt werden. Durch die enge Verzahnung mit dem Fachbereich Mathematik der Universität Kaiserslautern sowie durch zahlreiche Kooperationen mit internationalen Institutionen und Hochschulen in den Bereichen Ausbildung und Forschung ist ein großes Potenzial für Forschungsberichte vorhanden. In die Berichtreihe sollen sowohl hervorragende Diplom- und Projektarbeiten und Dissertationen als auch Forschungsberichte der Institutsmitarbeiter und Institutsgäste zu aktuellen Fragen der Techno- und Wirtschaftsmathematik aufgenommen werden.

Darüber hinaus bietet die Reihe ein Forum für die Berichterstattung über die zahlreichen Kooperationsprojekte des Instituts mit Partnern aus Industrie und Wirtschaft.

Berichterstattung heißt hier Dokumentation des Transfers aktueller Ergebnisse aus mathematischer Forschungs- und Entwicklungsarbeit in industrielle Anwendungen und Softwareprodukte - und umgekehrt, denn Probleme der Praxis generieren neue interessante mathematische Fragestellungen.

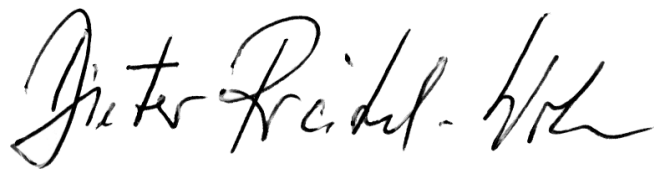

Prof. Dr. Dieter Prätzel-Wolters

Institutsleiter

Kaiserslautern, im Juni 2001 



\title{
MULTIBODY DYNAMICS SIMULATION OF GEOMETRICALLY EXACT COSSERAT RODS
}

\author{
Holger Lang ${ }^{\star}$, Joachim Linn ${ }^{\star}$ and Martin Arnold ${ }^{\dagger}$ \\ * Fraunhofer Institute for Industrial Mathematics \\ Fraunhofer Platz 1, 67663 Kaiserslautern, Germany \\ e-mail: holger.langeitwm. fraunhofer.de, \\ joachim.linnlitwm.fraunhofer.de \\ web page: http://www.itwm.fhg.de/de/mdf_employees_lang/lang/, \\ http://www.itwm.fhg.de/de/mdf_employees_linn/linn/ \\ $\dagger$ Institute for Mathematics, Martin-Luther-Universität Halle-Wittenberg \\ 06099 Halle (Saale), Germany \\ e-mail: martin.arnoldemathematik.uni-halle.de, \\ web page: http://www.mathematik.uni-halle.de/ arnold/
}

Keywords: Flexible multibody dynamics, Large deformations, Finite rotations, Constrained mechanical systems, Structural dynamics.

\begin{abstract}
In this paper, we present a viscoelastic rod model that is suitable for fast and sufficiently accurate dynamic simulations. It is based on Cosserat's geometrically exact theory of rods and is able to represent extension, shearing ('stiff' dof), bending and torsion ('soft' dof). For inner dissipation, a consistent damping potential from Antman is chosen. Our discrete model is based on a finite difference discretisation on a staggered grid. The right-hand side function $f$ and the Jacobian $\partial f / \partial(q, v, t)$ of the dynamical system $\dot{q}=v, \dot{v}=f(q, v, t)-$ after index reduction from three to zero - is free of higher algebraic (e.g. root) or transcendent (e.g. trigonometric or exponential) functions and is therefore cheap to evaluate. For the time integration of the system, we use well established stiff solvers like RADAU5 or DASPK. As our model yields computation times within milliseconds, it is suitable for interactive manipulation in 'virtual reality' applications. In contrast to fast common VR rod models, our model reflects the structural mechanics solutions sufficiently correct, as comparison with ABAQUS finite element results shows.
\end{abstract}




\section{INTRODUCTION}

The Cosserat rod model [1, 2, 3, 45, 46, 47] is an appropriate model for the geometrically exact simulation of deformable rods - i. e. slender one dimensional flexible structures - in space (statics) or space-time (quasistatics or dynamics). A Cosserat rod can be considered as the geometrically nonlinear generalisation of a Timoshenko-Reissner beam. In contrast to a Kirchhoff rod, which can be considered as a geometrically nonlinear generalisation of a Euler-Bernoulli beam, a Cosserat rod allows to model not only bending and torsion - these are 'soft dof' -, but as well extension and shearing - these are 'stiff dof'. For the Cosserat rod, like for the Kirchhoff rod, the overall deformation as response to moderate external loads, i. e. displacements, forces or moments, may become large, although locally the stresses and strains remain small. We remark that the Cosserat/Kirchhoff models are only 'skeleton' models. The reconstruction of the three dimensional displacement, stress and strain distributions through the rod cross sections can be conveniently carried out in a postprocessing by the use of 'warping functions' [20, 32].

This article is concerned with a discrete finite difference model of a Cosserat rod that is firmly based on structural mechanics and applicable to compute dynamical deformations very fast at sufficient accuracy. We continue the work [29, 30] from the ECCOMAS 2007, which considered the fast simulation of geometrically exact Kirchhoff rods in the quasistatic case.

The paper is structured as follows.

In section 2, we describe the basic equations for a Cosserat rod in the continuum, where we parametrise the rod directly with quaternions. Unit quaternions in the subgroup $\mathbb{S}^{3}=\partial B_{1}^{\mathbb{H}}(0)=$ $\{p \in \mathbb{H}:\|p\|=1\} \subset \mathbb{H}$ are an appropriate way to describe (non-commutative spatial) rotations in $S O(3)=\left\{Q \in \mathbb{R}^{3 \times 3}: Q Q^{T}=Q^{T} Q=I\right.$, $\left.\operatorname{det} Q=1\right\}$ for our purposes. This is analogous to unit complex numbers in the subgroup $\mathbb{S}^{1}=\partial B_{1}^{\mathbb{C}}(0)=\{z \in \mathbb{C}:\|z\|=1\} \subset \mathbb{C}$, which describe (commutative plane) rotations in $S O(2)=\left\{Q \in \mathbb{R}^{2 \times 2}: Q Q^{T}=Q^{T} Q=I\right.$, $\operatorname{det} Q=$ $1\}$. Of course, other possibilities, such as Rodriguez parameters, rotation vectors, Euler or Cardan angles, exist [?, 18, 36, 41]. All of them have their pros and cons. So as a pro, gimbal locking can be avoided with quaternions. A con is that they must be kept at unity length.

In section 3, we present our discrete numerical model of the Cosserat rod, based on finite differences. In contrast to the finite element method, where the primary unknowns are situated at the nodes (= vertices), we suggest a staggered grid discretisation. This means that the translatory resp. the rotatory dof are ordered in an alternating fashion and that the trapezoidal resp. midpoint quadrature formulas form the basis for the approximation of the internal energy integrals. This construction allows to interpret the discrete Cosserat rod as a sequence of - almost rigid - cylinders with the primary rotatory dof situated at the cylinder centers. These cylinders are connected with appropriate springs and dampers. Here the 'springs' and 'dampers' are seriously derived from the continuous Cosserat strain and curvature relations. Whereas the extensional and shearing strains, which belong to such cylinders, are discretised via finite differences in the one and only one canonical standard fashion, several choices for the discrete bending and torsion curvature measures are possible. These curvatures belong to the vertices between the cylinders and are the results of more or less accurate and expensive rotation interpolations. We formulate the final discrete model as a constrained mechanical system, resulting in the well known Lagrangian DAE system of index three. Index reduction to zero plus introduction of Baumgarte penalty accelerations gives a universal ODE formulation, suitable for any ODE solver. As an alternative to the Baumgarte method, stabilisation by projection is as well convenient and cheap. For our approach, the inverse mass-constraint matrix is expli- 
citly known and multiplication with the latter is exactly as expensive as multiplication with the mass-constraint matrix itself. The model can be implemented trigonometry and square root free, resulting in extremely cheap right hand side function and Jacobian evaluations. The extensible and inextensible Kirchhoff rod models can as well be conveniently fed into this framework.

In section 4, we present accurate numerical results, compared to ABAQUS finite element solutions. For bending and torsion scenarios e.g. in robotics or path planning simulations, we propose to impose strong damping on extension and shearing, which are of subordinate importance, and to solve the resulting stiff system via well established methods [22, 23, 35]. As our model allows computations within milliseconds, it is adequate for multibody dynamics simulations, especially for interactive manipulation applications.

Where can this work be situated within the state of the art rod models?

The handling of flexible objects in multibody dynamics simulations has been a long term field of research until today [5, 6, 7, 39, 42, 43, 44]. The standard approach, which is supported by today's commercial software packages such as SIMPACK, ADAMS or VIRTUALLAB, represents flexible structures by vibrational modes, e.g. of Craig and Bampton type [12], that are obtained from numerical modal analysis within the range of linear elasticity. Such methods are suitable and accurate to represent oscillatory response that results from linear response of the flexible structure. Unlike that, our approach in not of modal kind.

Our approach stands in contrast to the usual way in computational continuum mechanics, where the finite element approach is favored [6, 7, ?, ?, 14, 15, 20, 25, 26, 45, 46]. The reason for that is, that the main focus in FE is accuracy, not computational efficiency. The general problem in geometrically nonlinear FE is the proper interpolation of the finite rotations [38] such that objectivity of the strain measures is maintained, i. e. invariance under rigid body motions. This results in extremely technical and sophisticated models with expensive right hand side functions and Jacobians. Our approach is much simpler and objective is maintained by construction.

The main focus for rod models in the physics based modeling/virtual reality / computer graphics community is the numerical performance, rather than physical accuracy. Usually these models are too far away from continuum mechanics [48]. '... It's good enough if it looks good ...' and '... fooling the eye ...' is the main issue [37]. In our opinion, the best approach towards a physically serious rod model is the recent work [47]. However, here they did not publish total computational times.

\section{GEOMETRICALLY EXACT COSSERAT RODS IN THE CONTINUUM}

Our starting point for the continuous Cosserat rod model is the exposition in [45, 46]. For the constitutive material behaviour, we choose a simple linear viscoelastic one [1, 2, 3]. The elastic parameters can be straightforwardly deduced from material and geometric parameters [32]. Concerning the damping model, we note that it is macroscopic and phenomenological, it comprises not only pure material damping, but also miscellaneous damping mechanisms. We assume both the elastic and viscoelastic parts as diagonal. The generalisation to non-diagonal, symmetric and positively definite constitutive Hookean tensors or to nonlinear hyperelastic materials is straightforward and does not cause any harm in principle. We concentrate on the description of the internal potential, dissipation and kinetic energies in sections 2.1, 2.2 and 2.3 respectively, as these will be the basis for the discrete model later. In section 2.4 we give a detailed exposition of the dynamic equations of motion with the rotatory part in quaternion language. 
We start with the kinematics for the Cosserat rod. The Cosserat rod is completely determined by its centerline of mass centroids

$$
x:[0, L] \times[0, T] \rightarrow \mathbb{R}^{3}, \quad(s, t) \mapsto x(s, t)
$$

and its unit quaternion field

$$
p:[0, L] \times[0, T] \rightarrow \mathbb{S}^{3}=\partial B_{1}^{\mathbb{H}}(0) \hookrightarrow \mathbb{H}, \quad(s, t) \mapsto p(s, t) .
$$

The quaternion field uniquely determines its orthonormal frame field

$$
R \circ p:[0, L] \times[0, T] \stackrel{p}{\longrightarrow} \mathbb{S}^{3}=\partial B_{1}^{\mathbb{H}}(0) \stackrel{R}{\longrightarrow} S O(3), \quad(s, t) \mapsto R(p(s, t)) .
$$

The situation is depicted in Figure 1. Any point of the deformed rod in space $s$ and time $t$ is addressed by the deformation map

$$
[0, L] \times[0, T] \times \mathcal{A} \ni\left(s, t,\left(\xi_{1}, \xi_{2}\right)\right) \mapsto x(s, t)+\xi_{1} d^{1}(p(s, t))+\xi_{2} d^{2}(p(s, t)) .
$$

The parameter $s \in[0, L]$ is the arc length of the undeformed rod centerline, $L>0$ is the total undeformed arc length and $\mathcal{A} \subset \mathbb{R}^{2}$ is a bounded, connected coordinate domain for the coordinates $\left(\xi_{1}, \xi_{2}\right)$ in the cross section, which is assumed rigid and plane throughout the deformation. In classical differential geometry, the object $(x(\cdot, t),(R \circ p)(\cdot, t))$ constitutes a so-called 'framed curve'. For a quaternion $p=p_{0}+\hat{p}=\Re(p)+\Im(p)=\left(p_{0} ; p_{1}, p_{2}, p_{3}\right)^{T} \in \mathbb{H}$ the frame $R(p)$ is given by the Euler map

$$
R: \mathbb{H} \rightarrow \mathbb{R} S O(3), \quad p \mapsto\left(d^{1}(p)\left|d^{2}(p)\right| d^{3}(p)\right)=\left(2 p_{0}^{2}-\|p\|^{2}\right) \mathcal{I}+2 \hat{p} \otimes \hat{p}+2 p_{0} \mathcal{E}(\hat{p})
$$

with the alternating skew tensor $\mathcal{E}$, which identifies skew tensors in $s o(3)$ with their corresponding axial vectors in $\mathbb{R}^{3}$ via

$$
\mathcal{E}: \mathbb{R}^{3}=\Im(\mathbb{H}) \rightarrow s o(3), \quad \mathcal{E}(u) v=u \times v \quad \text { for } u, v \in \mathbb{R}^{3} .
$$

We write $u \simeq \mathcal{E}(u)$ for $u \in \mathbb{R}^{3}$. It is convenient to identify $\Im(\mathbb{H})=\mathbb{R}^{3}$. The directors $d^{1}(p)$ and $d^{2}(p)$ span the rigid cross section of the rod. The third director $d^{3}(p)$ is always normal to the cross section and is kept close to the tangent $\partial_{s} x$ of the centerline by stiff shearing springs. It holds that $R(\lambda p)=\lambda^{2} R(p)$ for each $p \in \mathbb{H}$ and $\lambda \in \mathbb{R}$. This property makes $R$ sensitive with respect to stretching of $p$. Especially, $R$ maps $\mathbb{S}^{3}$ into $S O(3)$. It holds $R(-p)=R(p)$, which implies that $p$ and its antipode $-p$ describe the same rotation. It is well known that for each rotation $Q$ in $S O(3)$ there exist exactly two unit quaternions - necessarily antipodes - that produce $Q$. Via $R$, the unit sphere $\mathbb{S}^{3}$ covers $S O(3)$ exactly two times [17, 19, 27]. Stretched rotation can be expressed via quaternions as $R(p) v=p v \bar{p}$ (forward) and $R(p)^{T} v=$ $\bar{p} v p$ (backward) for $p \in \mathbb{H}$ and $v \in \Im(\mathbb{H})=\mathbb{R}^{3}$, especially $d^{\nu}(p)=p e^{\nu} \bar{p}=R(p) e^{\nu}$ for each of the space fixed Euclidean base vectors $e^{1}, e^{2}$ and $e^{3}$ (classically denoted by ' $i$ ', ' $j$ ' and ' $k$ ') of $\Im(\mathbb{H})=\mathbb{R}^{3}$. Recall that the quaternion product is defined by

$$
p q=p_{0} q_{0}-\langle\hat{p}, \hat{q}\rangle+p_{0} \hat{q}+q_{0} \hat{p}+\hat{p} \times \hat{q} \quad \text { for } p, q \in \mathbb{H} .
$$

We use the symbols $p_{0}=\Re(p)$ resp. $\hat{p}=\Im(p)$ to denote the real resp. the imaginary (=vector) part and $\bar{p}=p_{0}-\hat{p}$ to denote the conjugate of a quaternion $p \in \mathbb{H}$. Note that $\bar{p}=\|p\|^{2} p^{-1}$, where $p^{-1}$ is the multiplicative inverse of $p$. Thus unit quaternions yield pure rotations without stretching. For details on the Hamilton quaternion division algebra, see [17].

Roughly speaking, the Cosserat rod possesses six degrees of freedom, three translatory and three rotatory ones. These equivalently correspond to the three strain and three curvature components. 


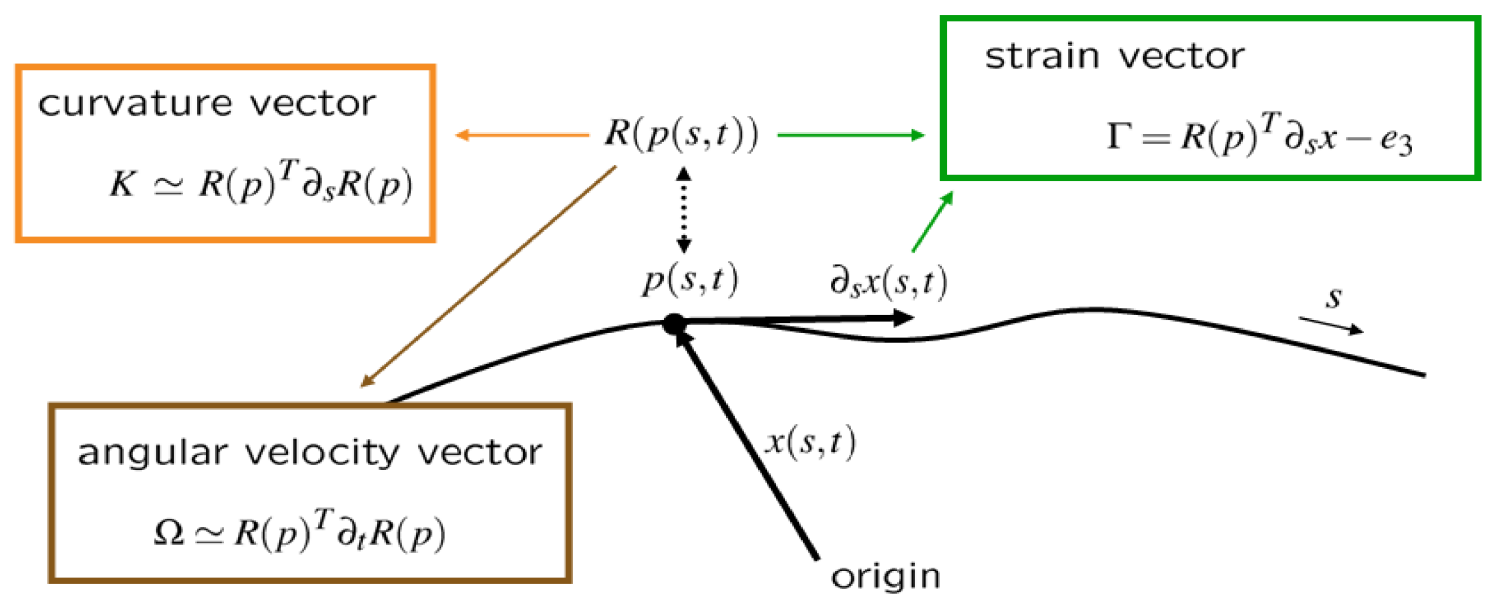

Figure 1: The continuous Cosserat rod

Remark 2.1 (Kirchhoff rod models) An 'extensible Kirchhoff rod' additionally satisfies the shearing constraints $\left\langle d^{1}, \partial_{s} x\right\rangle=\left\langle d^{2}, \partial_{s} x\right\rangle=0$, i. e. the cross sections stay always orthogonal to the centerline tangent. It therefore has only four dof. Extension is still allowed. An 'inextensible Kirchhoff rod' additionally satisfies $\left\|\partial_{s} x\right\|=1$, this means that the rod remains parametrised by arc length all the time during deformation. Then the centerline unit tangent $\tau=\partial_{s} x /\left\|\partial_{s} x\right\|$ is equal to $\partial_{s} x$, and the number of dof is equal to three.

\subsection{Continuous potential energies and strain measures}

The total potential energy $\mathcal{V}=\mathcal{V}_{S E}+\mathcal{V}_{B T}$ is additively decomposed into extensional and shearing energy $\mathcal{V}_{S E}$ and bending and torsion energy $\mathcal{V}_{B T}$. In $\mathcal{V}$, we include only the internal elastic energies, other conservative forces such as the gravitational force can simply be added as additional external forces. on the right hand side of the balance equations. The potential extensional and shearing energy is given by

$$
\mathcal{V}_{S E}=\frac{1}{2} \int_{0}^{L} \Gamma^{T} C^{\Gamma} \Gamma \mathrm{d} s, \quad C^{\Gamma}=\operatorname{diag}\left(G A_{1}, G A_{2}, E A\right),
$$

where the material strains are defined by

$$
\Gamma=R(p)^{T} \partial_{s} x-e^{3} .
$$

$\Gamma^{1}$ resp. $\Gamma^{2}$ are the strains corresponding to shearing in $d^{1}$ - resp. $d^{2}$-direction, $\Gamma^{3}$ is the strain corresponding to extension in $d^{3}$-direction. In components, we have

$$
\Gamma^{1}=\left\langle d^{1}(p), \partial_{s} x\right\rangle, \quad \Gamma^{2}=\left\langle d^{2}(p), \partial_{s} x\right\rangle, \quad \Gamma^{3}=\left\langle d^{3}(p), \partial_{s} x\right\rangle-1 .
$$

$E>0$ denotes Young's modulus and $G>0$ the shear modulus of the material, $A=\iint_{\mathcal{A}} \mathrm{d}\left(\xi_{1}, \xi_{2}\right)$ is the area of the rigid cross section, $A_{1}=\kappa_{1} A$ and $A_{2}=\kappa_{2} A$ are some effective cross section areas with some Timoshenko shear correction factors $0<\kappa_{1}, \kappa_{2} \leq 1$, cf. [11]. The potential bending and torsion energy is

$$
\mathcal{V}_{B T}=\frac{1}{2} \int_{0}^{L} K^{T} C^{K} K \mathrm{~d} s, \quad C^{K}=\operatorname{diag}\left(E I_{1}, E I_{2}, G J\right),
$$


where the material curvature vector (or the 'Darboux' vector) is given by

$$
K \simeq \mathcal{E}(K)=R(p)^{T} \partial_{s} R(p)
$$

$K^{1}$ resp. $K^{2}$ are the curvatures corresponding to bending around the $d^{1}$ - resp. $d^{2}$-axis, $K^{3}$ is the curvature corresponding to torsion around the $d^{3}$-axis. $I_{1}=\iint_{\mathcal{A}} \xi_{2}^{2} \mathrm{~d}\left(\xi_{1}, \xi_{2}\right)$ and $I_{2}=$ $\iint_{\mathcal{A}} \xi_{1}^{2} \mathrm{~d}\left(\xi_{1}, \xi_{2}\right)$ are the geometric moments of inertia of the rigid cross section. $J=I_{3}=$ $\iint_{A}\left(\xi_{1}^{2}+\xi_{2}^{2}\right) \mathrm{d}\left(\xi_{1}, \xi_{2}\right)=I_{1}+I_{2}$ denotes its polar moment. If the cross section is symmetric, then we have $I_{1}=I_{2}$ and $J=I_{3}=2 I_{1}=2 I_{2}$. The conservative elastic forces $F^{\Gamma}$ and moments $M^{K}$ are derived from the potential energy as $F^{\Gamma}=C^{\Gamma} \Gamma=\frac{1}{2} \partial_{\Gamma}\left(\Gamma^{T} C^{\Gamma} \Gamma\right)$ and $M^{K}=$ $C^{K} K=\frac{1}{2} \partial_{K}\left(K^{T} C^{K} K\right)$. Clearly, any other more general form of hyperelastic constitutive material behaviour could be used instead [45]. If the rod possesses non-vanishing precurvature $K^{0}:[0, L] \rightarrow \mathbb{R}^{3}$ in the undeformed configuration, $K$ can simply be replaced by $K-K^{0}$ in (3) throughout the model [45].

It is well known that the strain measures (2) and (4) are frame indifferent.

\subsection{Continuous dissipation energies and strain rates}

Dissipation is not explained in [45]. For our dissipation potential, we follow [1, 2, 3] and choose friction forces resp. moments that are proportional to the strain rates resp. curvature rates. The total dissipation energy $\mathcal{D}=\mathcal{D}_{S E}+\mathcal{D}_{B T}$ consists of dissipative extensional and shearing energy $\mathcal{D}_{S E}$ plus dissipative bending and torsion energy $\mathcal{D}_{B T}$. The dissipative extensional and shearing energy is

$$
\mathcal{D}_{S E}=\int_{0}^{L} \dot{\Gamma}^{T} C^{\dot{\Gamma}} \dot{\Gamma} \mathrm{d} s, \quad C^{\dot{\Gamma}}=\operatorname{diag}\left(c_{1}^{\dot{\Gamma}}, c_{2}^{\dot{\Gamma}}, c_{3}^{\dot{\Gamma}}\right)
$$

with the material strain rates $\dot{\Gamma}=\partial_{t} R(p)^{T} \partial_{s} x+R(p)^{T} \partial_{s t}^{2} x$. The dissipative bending and torsion energy is given by

$$
\mathcal{D}_{B T}=\int_{0}^{L} \dot{K}^{T} C^{\dot{K}} \dot{K} \mathrm{~d} s, \quad C^{\dot{K}}=\operatorname{diag}\left(c_{1}^{\dot{K}}, c_{2}^{\dot{K}}, c_{3}^{\dot{K}}\right)
$$

with the material curvature rates $\dot{K} \simeq \partial_{t} \mathcal{E}(K)=\partial_{t} R(p)^{T} \partial_{s} R(p)+R(p)^{T} \partial_{s t}^{2} R(p)$. The nonnegative constants $c_{\nu}^{\dot{\Gamma}}$ and $c_{\nu}^{\dot{K}}$ for $\nu=1,2,3$ denote some viscoelastic material parameters. The dissipative damping forces $F^{\dot{\Gamma}}$ and moments $M^{\dot{K}}$ are derived from the dissipation potential as $F^{\dot{\Gamma}}=2 C^{\dot{\Gamma}} \dot{\Gamma}=\partial_{\dot{\Gamma}}\left(\dot{\Gamma}^{T} C^{\dot{\Gamma}} \dot{\Gamma}\right)$ and $M^{\dot{K}}=2 C^{\dot{K}} \dot{K}=\partial_{\dot{K}}\left(\dot{K}^{T} C^{\dot{K}} \dot{K}\right)$. Of course, any other more general form of consistent viscoelastic constitutive material behaviour could replace these assumptions.

\subsection{Continuous kinetic energies}

The total kinetic energy $\mathcal{T}=\mathcal{T}_{T}+\mathcal{T}_{R}$ consists of two parts, the translatory $\mathcal{T}_{T}$ and the rotatory $\mathcal{T}_{R}$ kinetic energy, given by

$$
T_{T}=\frac{\varrho A}{2} \int_{0}^{L}\|\dot{x}\|^{2} \mathrm{~d} s, \quad T_{R}=\frac{\varrho}{2} \int_{0}^{L} \Omega^{T} I \Omega \mathrm{d} s, \quad I=\operatorname{diag}\left(I_{1}, I_{2}, J\right) .
$$

Here the material angular velocity vector (or the 'vorticity' vector) is

$$
\Omega \simeq \mathcal{E}(\Omega)=R(p)^{T} \partial_{t} R(p)
$$


Here $\varrho>0$ is the material density, $I_{1}, I_{2}$ and $J=I_{3}$ are as above, and we identify $\Omega \simeq \mathcal{E}(\Omega)$. We rewrite the rotatory kinetic energy with the identity $\Omega^{T} I \Omega=\dot{p}^{T} \mu(p) \dot{p}$ and the $p$ dependent $4 \times 4$ quaternion mass matrix [24]

$$
\mu(p)=4 \mathcal{Q}(p) I \mathcal{Q}(p)^{T}, \quad \mathcal{Q}(p)=\left(\begin{array}{c|c}
p_{0} & -\hat{p}^{T} \\
\hline \hat{p} & p_{0} \mathcal{I}+\mathcal{E}(\hat{p})
\end{array}\right) .
$$

The corresponding computations are carried out in detail in [41]. The mass matrix satisfies the symmetry property $\mu(-p)=\mu(p)$, which is a consequence of the fact that both $p$ and $-p$ describe the same rotation $R(p)=R(-p)$. The kernel of $\mu(p)$ is given by ker $\mu(p)=\mathbb{R} p$, consequently we have $\operatorname{rk} \mu(p)$ equal to three. $\mu(p)$ is positively semi-definite with the one singular dimension in direction $p$.

The reader should note that the situation for $K$ and $\Omega$ is completely symmetric from a two dimensional field view. For our discrete model, evolution (4) is the basis for spatial discretisation. Evolution (8) is solved 'continuously' in time, of course.

\subsection{Continuous equations of motion}

It can be shown from a two dimensional variational principle, that the Cosserat rod, for given exterior material force densities $\hat{F}=\hat{F}(t)$ (per length) and given exterior material moment densities $\hat{M}=\hat{M}(t)$ (per length), satisfies the following nonlinear system of partial differential equations

$$
\left\{\begin{aligned}
\varrho A \ddot{x} & =\partial_{s}(p F \bar{p})+p \hat{F} \bar{p} \\
\varrho\left[\mu \ddot{p}-\frac{1}{2} \partial_{p}\left(\dot{p}^{T} \mu \dot{p}\right)+\partial_{p}(\mu \dot{p}) \dot{p}\right] & =2 x^{\prime} p F+\partial_{s}(2 p M)+2 p^{\prime} M+2 p \hat{M}-\lambda p \\
0 & =\|p\|^{2}-1
\end{aligned}\right.
$$

where $^{\prime}=\partial_{s}$ and ${ }^{\cdot}=\partial_{t}$. The viscoelastic forces and moments are given by $F=C^{\Gamma} \Gamma+2 C^{\dot{\Gamma}} \dot{\Gamma}$ and $M=C^{K} K+2 C^{\dot{K}} \dot{K}$. Together with appropriate initial and boundary conditions, this is the system that has to be solved for the unknowns $x, p$ and $\lambda$.

Originally in [45, 46], averaging the normal Piola-Kirchhoff tractions and corresponding torques over the cross section of the deformed rod, it was shown that the Cosserat rod must satisfy the following form of the balance equations of motion, namely

$$
\left\{\begin{array}{rl}
\varrho A \ddot{x} & =\partial_{s} f+\hat{f} \\
\varrho(i \dot{\omega}+\omega \times i \omega) & =\partial_{s} m+\partial_{s} x \times f+\hat{m}
\end{array} .\right.
$$

This original formulation of the equations of motion, which is probably more familiar to the reader, can as well be found in [1, 2, 3]. Here the spatial quantities $\gamma=R \Gamma, k=R K$, $\omega=R \Omega, i=R I R^{T}, f=R F$ and $m=R M$ are obtained from the corresponding material ones by a push forward via $R$. The components of any of these spatial quantities in the moving coordinate system $\left(d^{1}, d^{2}, d^{3}\right)$ are identical to the components of the corresponding material quantities, measured in the fixed global coordinate system $\left(e^{1}, e^{2}, e^{3}\right)$.

It can be shown that the systems (10) and (11) are equivalent.

\section{DISCRETE COSSERAT RODS VIA FINITE DIFFERENCES}

Here we present our discrete version of the Cosserat model. Sections $3.1, \ldots, 3.4$ are the discrete counterparts of sections 2.1 ..., 2.4 respectively. In contrast to finite element methods, 
we propose the following staggered grid discretisation. We subdivide the arc length interval $[0, L]$ into $N$ segments $\left[s_{n-1}, s_{n}\right]$ with the vertices

$$
0=s_{0}<s_{1}<\ldots<s_{N-1}<s_{N}=L .
$$

Together with the midpoints $s_{n-1 / 2}=\left(s_{n-1}+s_{n}\right) / 2$, we have the staggered grid

$$
0=s_{0}<s_{1 / 2}<s_{1}<\ldots<s_{N-1}<s_{N-1 / 2}<s_{N}=L .
$$

Now we let the discrete translatory degrees of freedom $x_{n}:[0, T] \rightarrow \mathbb{R}^{3}$, i.e. the cross section centroids, live on the vertices,

$$
x_{0}(\cdot) \approx x\left(s_{0}, \cdot\right), \quad \ldots, \quad x_{N}(\cdot) \approx x\left(s_{N}, \cdot\right),
$$

and the discrete rotatory degrees of freedom $p_{n-1 / 2}:[0, T] \rightarrow \mathbb{H}$, i. e. the quaternions specifying the frame orientations, on the segment midpoints,

$$
p_{1 / 2}(\cdot) \approx p\left(s_{1 / 2}, \cdot\right), \quad \ldots, \quad p_{N-1 / 2}(\cdot) \approx p\left(s_{N-1 / 2}, \cdot\right) .
$$

In order that the quaternions remain in $\mathbb{S}^{3}$, we have to introduce the discrete Lagrange multipliers $\lambda_{n-1 / 2}:[0, T] \rightarrow \mathbb{R}$ of dimension 'energy', situated on the midpoints,

$$
\lambda_{1 / 2}(\cdot) \approx \lambda\left(s_{1 / 2}, \cdot\right), \quad \ldots, \quad \lambda_{N-1 / 2}(\cdot) \approx \lambda\left(s_{N-1 / 2}, \cdot\right)
$$

and $N$ constraints $2 g_{n-1 / 2}(q)=\left\|p_{n-1 / 2}\right\|^{2}-1$, which are situated here as well. The corresponding frames $R\left(p_{n-1 / 2}\right)$ and the directors $d^{\nu}\left(p_{n-1 / 2}\right)$ live as well on the midpoints.

A staggered grid discretisation was already proposed in [16, 28] for the case of an inextensible Kirchhoff rod. We extend this idea to the shear and extensional flexible Cosserat rod with quaternions. The staggered grid approach allows it to interpret the rod as a sequence of almost rigid cylinders, connected with appropriate 'bushings' for bending and torsion. We consequently use the notation ${ }_{n}$ for quantities at the vertices $s_{n}$; here $n$ ranges from 0 to $N$. We use the notation ${ }_{n-1 / 2}$ for quantities that are situated on the midpoints $s_{n-1 / 2}$; here $n$ ranges from 1 to $N$, if not otherwise explicitly stated. The situation is depicted in Figure 2 ,

In the sequel, we discretise the continuous internal Cosserat energy integrals $\mathcal{V}, \mathcal{T}$ and $\mathcal{D}$ by the use of either midpoint or trapezoidal quadrature, depending on where which magnitude is 'at home'. The weight factors for the midpoint rule are the segment lengths $\Delta s_{n-1 / 2}=$ $s_{n}-s_{n-1}$. Likewise, the weights for the trapezoidal rule are the lengths of the bucked segments $2 \delta s_{0}=\Delta s_{1 / 2}, 2 \delta s_{n}=\Delta s_{n-1 / 2}+\Delta s_{n+1 / 2}$ and $2 \delta s_{N}=\Delta s_{N-1 / 2}$. Then, with $q=(x, p)$, the discrete potential energy $\mathcal{V}$, the discrete kinetic energy $\mathcal{T}$, the discrete dissipation energy $\mathcal{D}$, the Lagrangian function $\mathcal{L}=\mathcal{T}-\mathcal{V}-g^{T} \lambda$ and exterior forces $\psi(t)$, the variational principle

$$
\delta \int_{0}^{T} \mathcal{L} \mathrm{d} t-\int_{0}^{T} \partial_{\dot{q}} \mathcal{D} \delta q \mathrm{~d} t+\int_{0}^{T} \psi \delta q \mathrm{~d} t=0
$$

yields the Euler-Lagrange equations as the well-known index three differential algebraic system of equations

$$
\left\{\begin{aligned}
\mathcal{M}(q) \ddot{q} & =\mathcal{F}(q, \dot{q}, t)-\mathcal{G}(q)^{T} \lambda \\
0 & =g(q)
\end{aligned}\right.
$$

with the right hand side forces given by $\mathcal{F}(q, \dot{q}, t)=\psi(t)-\partial_{q} \mathcal{V}-\partial_{\dot{q}} \mathcal{D}+\partial_{q} \mathcal{T}-\partial_{q}(\mathcal{M}(q) \dot{q}) \dot{q}$, cf. [4, 23]. 


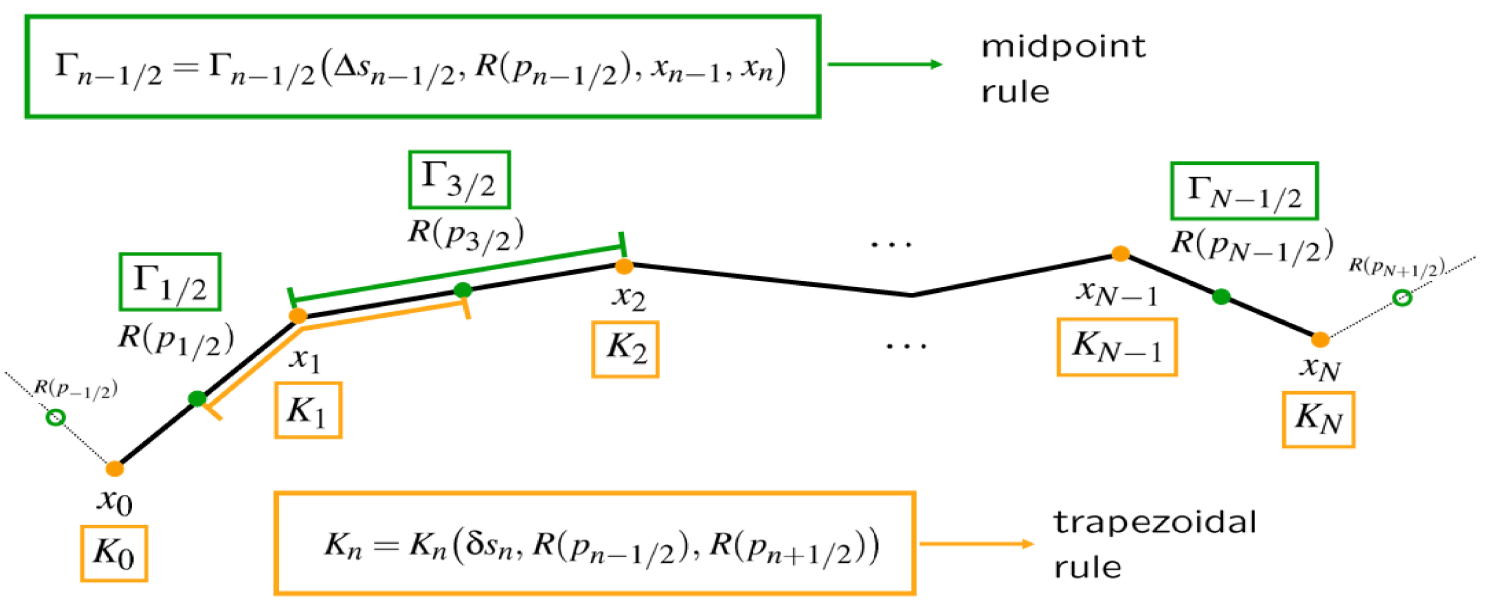

Figure 2: Our discrete Cosserat rod

How to solve the dynamical equations? One method is to use eight local charts $p_{k}=$ $\pm\left(1-\sum_{j \neq k} p_{j}^{2}\right)^{1 / 2}$ for $k=0, \ldots, 3$ that cover the unit sphere $\mathbb{S}^{3}=\partial B_{1}^{\mathbb{H}}(0) \subset \mathbb{H}$. Numerical approaches using local charts exist [23], however, changing charts is a tedious task and it is much easier to formulate the equations of motion as a system of differential algebraic equations, where we keep the quaternion unity condition $2 g=\|p\|^{2}-1=0$ as a hard algebraic constraint. It is well known that the numerical solution of the index three system involves difficulties such as poor convergence of Newton's method [4, 18, 21, 23, 44], so we reduce the index to one

$$
\left(\begin{array}{c}
\ddot{q} \\
\lambda
\end{array}\right)=\left(\begin{array}{cc}
\mathcal{M}(q) & \mathcal{G}(q)^{T} \\
\mathcal{G}(q) & 0
\end{array}\right)^{-1}\left(\begin{array}{c}
\mathcal{F}(q, \dot{q}, t) \\
-g_{q}^{\prime \prime}(\dot{q}, \dot{q})
\end{array}\right)
$$

and solve the index zero subsystem $\ddot{q}=\ddot{q}(q, \dot{q}, t)$. Stabilisation of the quaternion unity constraints in the index one/zero case is especially easy and cheap.

Remark 3.1 (Boundary conditions) In order to apply clamped boundary rotations properly, i. e. $p_{0}=p_{0}(t)$ and $p_{N}=p_{N}(t)$, we introduce virtual ghost quaternions $p_{-1 / 2}$ and $p_{N+1 / 2}$, which is a standard technique [33]. They are situated beyond the boundary and defined as the spherical linear extrapolation of $p_{1 / 2}$ via $p_{0}(t)$ or $p_{N-1 / 2}$ via $p_{N}(t)$ respectively. The handling of free boundary conditions with vanishing boundary curvature and moment is clear.

\subsection{Discrete potential energies and strain measures}

We start with the discrete version of the potential extensional and shearing energy. The strains $\Gamma_{n-1 / 2}$ are situated on the midpoints. Therefore, the integral (1) is approximated with the midpoint rule,

$$
\mathcal{V}_{S E}=\frac{1}{2} \sum_{n=1}^{N} \Delta s_{n-1 / 2} \Gamma_{n-1 / 2}^{T} C^{\Gamma} \Gamma_{n-1 / 2}, \quad \Gamma_{n-1 / 2}=R\left(p_{n-1 / 2}\right)^{T} \frac{\Delta x_{n-1 / 2}}{\Delta s_{n-1 / 2}}-e^{3},
$$

where $\Gamma_{n-1 / 2}$ denote the discrete material strains. They locally depend on $x_{n-1}, x_{n}$ and $p_{n-1 / 2}$, see Figure 2. Of course, finite difference schemes of higher order are possible [31].

We continue with the discrete version of the potential bending and torsion energy. The curvatures $K_{n}$ are situated on the vertices. Consequently, we approximate (3) with the trapezoidal 
rule

$$
\mathcal{V}_{B T}=\frac{1}{2} \sum_{n=0}^{N} \delta s_{n} K_{n}^{T} C^{K} K_{n}, \quad K_{n}=K_{n}\left(\delta s_{n}, R\left(p_{n-1 / 2}\right), R\left(p_{n+1 / 2}\right)\right),
$$

where the discrete material curvatures $K_{n}$ depend on $R\left(p_{n-1 / 2}\right)$ and $R\left(p_{n+1 / 2}\right)$, see Figure 2 , Frame indifference requires that $K_{n}$ is in fact a function of $R\left(p_{n-1 / 2}\right)^{T} R\left(p_{n+1 / 2}\right)$. And again it is clear that higher order finite difference approximations are possible [31].

The question arises, how to interpolate the two quaternions $p_{n-1 / 2}$ resp. $p_{n+1 / 2}$, living on the segment midpoints $s_{n-1 / 2}$ resp. $s_{n+1 / 2}$, to a quaternion $p_{n}$, living on the vertex at $s_{n}$, and to choose an appropriate difference expression in order to evaluate $\mathcal{E}(K)=R(p)^{T} \partial_{s} R(p)$ at the vertex $s_{n}$. So for example, the simple ansatz in [47] chooses linear midpoint interpolation $p_{n}=\left(p_{n-1 / 2}+p_{n+1 / 2}\right) / 2$, the secant $\delta p_{n}=p_{n+1 / 2}-p_{n-1 / 2}$ and $\delta s_{n}=s_{n+1 / 2}-s_{n-1 / 2}$, irregardless of the fact that $p_{n}$ violates the unity constraint. This results in an expression for $K_{n}$ that is proportional to $\Im\left(\bar{p}_{n-1 / 2} p_{n+1 / 2}\right)$, which is non-physically soft and suffers from very poor stability properties for large bending or torsion angles. Phenomena such as 'quaternion flipping' are the consequence.

The details of more sophisticated approaches go beyond the scope of the present paper. For thorough discussions, how to interpolate the rotations, we refer to [38].

\subsection{Discrete dissipation energies and strain rates}

The discretisation of the dissipation potential has to be consistent with the discretisation of the potential energies. We start with the dissipative extensional and shearing energy. The strain rates $\dot{\Gamma}_{n-1 / 2}$ are situated at the midpoints. Thus, the continuous integral (5) is approximated with the midpoint rule,

$$
\mathcal{D}_{S E}=\sum_{n=1}^{N} \Delta s_{n-1 / 2} \dot{\Gamma}_{n-1 / 2}^{T} C^{\dot{\Gamma}} \dot{\Gamma}_{n-1 / 2}, \quad \dot{\Gamma}_{n-1 / 2}=\frac{\partial}{\partial t} \Gamma_{n-1 / 2}
$$

where the discrete material strain rates are given by the time derivative of (13). They depend both on the positions $x_{n-1}, x_{n}, p_{n-1 / 2}$ and the velocities $\dot{x}_{n-1}, \dot{x}_{n}, \dot{p}_{n-1 / 2}$. Concerning the discrete dissipative bending and torsion energy, the curvature rates $\dot{K}_{n}$ are situated - like the curvatures themselves - on the vertices. Thus, (6) is approximated with a trapezoidal sum,

$$
\mathcal{D}_{B T}=\sum_{n=0}^{N} \delta s_{n} \dot{K}_{n}^{T} C^{\dot{K}} \dot{K}_{n}, \quad \dot{K}_{n}=\frac{\partial}{\partial t} K_{n},
$$

where the discrete material curvature rates are the time derivative of (14), depending on $p_{n-1 / 2}$, $p_{n+1 / 2}$ and $\dot{p}_{n-1 / 2}, \dot{p}_{n+1 / 2}$.

\subsection{Discrete kinetic energies}

As the centroids $x_{n}$ are situated at the vertices $s_{n}$, we discretise the translatory kinetic energy integral in (7) by the trapezoidal rule and, as the quaternions are situated on the midpoints $s_{n-1 / 2}$, the rotatory kinetic energy integral (7) is approximated with the midpoint rule

$$
\mathcal{T}_{T}=\frac{\varrho A}{2} \sum_{n=0}^{N} \delta s_{n}\left\|\dot{x}_{n}\right\|^{2}, \quad \mathcal{T}_{R}=\frac{\varrho}{2} \sum_{n=1}^{N} \Delta s_{n-1 / 2} \dot{p}_{n-1 / 2}^{T} \mu\left(p_{n-1 / 2}\right) \dot{p}_{n-1 / 2} .
$$


Thus, the translatory mass is situated at the vertices and the rotatory quaternion mass belongs to the segments. The discrete material angular velocities $\mathcal{E}\left(\Omega_{n-1 / 2}\right)=R\left(p_{n-1 / 2}\right)^{T} \partial_{t} R\left(p_{n-1 / 2}\right)$ as well belong to the midpoints. The mass matrix $\mathcal{M}(q)$ of the system is block diagonal with alternating $3 \times 3$ (translatory, diagonal and constant) and $4 \times 4$ (rotatory, position-dependent) blocks.

Each summand in (15) can be interpreted as the rotatory energy of a rigid body with moments of inertia equal to $\bar{I}^{1}=\varrho \Delta s I_{1}, \bar{I}^{2}=\varrho \Delta s I_{2}$ and $\bar{I}^{3}=\varrho \Delta s I_{3}$, see [36, 41]. These are in fact the physical moments of inertia of a disc with vanishing thickness. Now we consider the rod as decomposed into $N$-almost rigid - cylinders with moments of inertia, which we denote by $I^{1}$, $I^{2}$ and $I^{3}$. Comparison shows that $I^{\nu}-\bar{I}^{\nu}=\mathcal{O}\left(\Delta s^{3}\right)$ for $\nu=1,2$, and $I^{3}-\bar{I}^{3}=0$. For fine discretisations, these defects may be neglected. Otherwise, a more detailed analysis might be necessary.

For each centroid $x_{n}$ with mass $\varrho A \delta s_{n}$, the translatory mass-matrix block is given by a $3 \times 3$ diagonal, constant, state independent block $\varrho A \delta s_{n} \mathcal{I}$. For the rotatory part, we fix a segment $\Delta s=\Delta s_{n-1 / 2}$ and its quaternion $p=p_{n-1 / 2}$. The constraints of position, velocity and acceleration are written $2 g=\|p\|^{2}-1=0, \dot{g}=G(p) \dot{p}=\langle p, \dot{p}\rangle=0$ and $\ddot{g}=G(p) \ddot{p}+g_{p}^{\prime \prime}(\dot{p}, \dot{p})=$ $\langle p, \ddot{p}\rangle+\|\dot{p}\|^{2}=0$ respectively, where $G(p)=\partial_{p} g(p)=p^{T}$. Thus, the rotatory quaternion massconstraint-matrix $5 \times 5$ block is given by

$$
\left(\begin{array}{c|c}
\varrho \Delta s \mu(p) & G(p)^{T} \\
\hline G(p) & 0
\end{array}\right)=\left(\begin{array}{c|c}
\varrho \Delta s \mu(p) & p \\
\hline p^{T} & 0
\end{array}\right)
$$

with the singular quaternion mass $\mu(p)$ from (9). The inverse of (16) exists iff $p \neq 0$ and can be explicitly algebraically computed. It has exactly the same structure as (16) and can therefore be calculated at exactly the same numerical cost.

The question arises, why to use $p_{n-1 / 2}$ and $\dot{p}_{n-1 / 2}$ as the primary unknowns and not $p_{n-1 / 2}$ and $\Omega_{n-1 / 2}$, as it is frequently recommended [41, 47]. The reason is that many standard ODE or DAE solvers such as RADAU5, SEULEX or RODAS [21, 23, 34] support sparse / banded linear algebra that is specially adapted to second order systems of the form $\dot{q}=v, \dot{v}=\dot{v}(q, v, t)$.

Remark 3.2 (Condition) It can be shown that the condition number of the mass-constraint matrix (16) is equal to the ratio $\max M / \min M$, where $M=\left\{\varrho \Delta s I_{1}, \varrho \Delta s I_{2}, \varrho \Delta s I_{3}, 1 / 4\right\}$. So, for typical material parameters and discretisations, the system is rather ill-conditioned.

'In the case of ill-conditioned linear systems, scaling of the constraints ... should be tried.' [44].

By scaling the constraint equation by a factor of $c>0$, the condition number of any quaternion mass-constraint block (16) can be influenced. If $g$ is replaced by $c g, G$ has to be replaced by $c G$ and $g_{p}^{\prime \prime}$ by $c g_{p}^{\prime \prime}$. For the special case of symmetric cross sections for example, where $I_{1}=I_{2}=I$, $J=I_{1}+I_{2}=2 I$, any choice $c \in 4 \varrho \Delta s[I, J]$ leads to a condition number of two. Note that the Lagrange multiplier $\lambda$ scales with $1 / c$, so that the constraint force $G^{T} \lambda$, which is normal to the unit sphere in accordance to d'Alembert's principle and keeps the quaternions on its $\mathbb{S}^{3}$ orbit, remains unchanged.

For the index zero/one formulation (12), the position $q$ drifts quadratically from the constraint manifold $\{q: g(q)=0\}$. Subsequent projection $p \mapsto p /\|p\|$ of the quaternion position and $\dot{p} \mapsto \dot{p}-\langle p, \dot{p}\rangle p$ of the quaternion velocity is especially cheap, it may be applied even after each successful integration step. However, easy and efficient implementations of this method are 
restricted to one step integration methods, excluding BDF methods with order of at least two. Another stabilisation technique - already on the model level - is the Baumgarte method [23]. Imposing the linear combinations $\ddot{g}+2 r \dot{g}+\omega^{2} g=0$ as constraints with $\omega, r>0$, the index one Baumgarte formulation is obtained. The equation for the Lagrange multiplier $\lambda$ remains unchanged. The additional penalty accelerations are needed to pull $p$ back to $\mathbb{S}^{3}$, if the velocity constraint $\langle p, \dot{p}\rangle \neq 0$ or the position constraint $\|p\| \neq 1$ is violated. The choice $r=\omega$ leads to critical damping of the constraint defect $g$. One might argue that the Baumgarte method is energy dissipating. This is true, but the projection method is as well, see [23, 24]. Another frequent objection is that the Baumgarte method introduces additional stiffness into the system. This is true, but the Cosserat shearing and extensional 'springs' are already stiff, so that this does not mean a drawback from the practical viewpoint.

\subsection{Discrete equations of motion}

Carrying out the details of the preceding sections, adding exterior material force densities $\hat{F}$. $(t)$ and exterior material moment densities $\hat{M} .(t)$ and plugging all the pieces together, we obtain the discrete translatory and rotatory balance equations in index one formulation. Letting $n=0, \ldots, N$ and $\nu=1 / 2, \ldots, N-1 / 2$, the topological structure of the system is seen to be

$$
\left\{\begin{array}{l}
\ddot{x}_{n}=\ddot{x}_{n}\left(x_{n-1}, p_{n-\frac{1}{2}}, x_{n}, p_{n+\frac{1}{2}}, x_{n+1} ; \dot{x}_{n-1}, \dot{p}_{n-\frac{1}{2}}, \dot{x}_{n}, \dot{p}_{n+\frac{1}{2}}, \dot{x}_{n+1} ; t\right) \\
\ddot{p}_{\nu}=\ddot{p}_{\nu}\left(p_{\nu-1}, x_{\nu-\frac{1}{2}}, p_{\nu}, x_{\nu+\frac{1}{2}}, p_{\nu+1} ; \dot{p}_{\nu-1}, \dot{x}_{\nu-\frac{1}{2}}, \dot{p}_{\nu}, \dot{x}_{\nu+\frac{1}{2}}, \dot{p}_{\nu+1} ; t\right) \\
\lambda_{\nu}=\lambda_{\nu}\left(p_{\nu-1}, x_{\nu-\frac{1}{2}}, p_{\nu}, x_{\nu+\frac{1}{2}}, p_{\nu+1} ; \dot{p}_{\nu-1}, \dot{x}_{\nu-\frac{1}{2}}, \dot{p}_{\nu}, \dot{x}_{\nu+\frac{1}{2}}, \dot{p}_{\nu+1} ; t\right)
\end{array}\right.
$$

This results in upper and lower bandwidths of the Jacobians $\partial \ddot{q} / \partial q$ and $\partial \ddot{q} / \partial \dot{q}$ equal to ten. The corresponding index zero subsystem $\dot{u}=f(u, t), u=(q, v), q=(x, p), \dot{q}=v$, which is obtained by discarding the equation for the multipliers, can be solved by any ODE integrator.

It can be shown that our approach yields consistent discretisations of (10).

The reader should observe that, intrinsically in the model, there are many common terms and (skew-)symmetries. This is one reason, why the model can be implemented with very few arithmetic operations. So, the model needs about $174 N$ additions, $111 N$ subtractions, $289 N$ multiplications and $3 N$ divisions, where $N$ is the number of segments. All in all, the evaluation cost for $f$ and $\partial f / \partial u$ is thus extremely small.

Remark 3.3 (Kirchhoff rod models) Usually in robotics applications for example, one is interested solely in the proper modeling of bending and torsion. The stiff extensional and shearing springs only deteriorate the computational performance.

\section{'If stiff components are not really necessary, avoid them by model reduction.' [44]}

The (in)extensible Kirchhoff rods can be incorporated easily by the introduction of additional constraints $g_{n-1 / 2}^{\nu}=\Gamma_{n-1 / 2}^{\nu}, \nu=1,2(, 3)$. For the boundary conditions, the handling of $t$ dependent constraints is explained in [4]. The advantage is, that for coarse discretisations, a significant increase in step size can be achieved. But the approach has some disadvantages. The first disadvantage is that an easy ODE formulation $\dot{u}=f(t, u)$ is problematic, since the inverse of the mass-constraint matrix is full, so that an $\mathcal{O}(N)$ multibody formalism [18, 40] has to be applied. The second is that stabilisation of the constraints is not trivial and numerically more expensive as it is for our discrete Cosserat model. The third concerns the inextensible Kirchhoff rod model, which allows the largest step sizes: The mass-constraint matrix becomes singular, 
for example when the rod is straight. (This is often the initial configuration!) Therefore, additional inconvenient treatments are necessary. To sum up, in the treatment of the stiff extensional and shearing springs, we rather follow another advise,

\section{'Dissipation in the elastic model alleviates the numerical difficulties ... .' [44].}

Our tests yield that the full Cosserat rod model with consistent strong damping on tension and shearing performs better than the (in)extensible Kirchhoff one, if the constraints are introduced as above. However, a pure bending and torsion model in quaternion minimal coordinates will be the topic of further research.

\section{NUMERICAL EXAMPLES}

In this section, we compare the solutions of our model to finite element solutions, computed with ABAQUS both $1 \mathrm{D}$ beam and 3D continuum elements. We further demonstrate the performance of our model for two simple scenarios with strong damping on the extension and shearing dof.

Test 1 vs. ABAQUS (dynamic) In a first test, we compare a $L=1 \mathrm{~m}$ long dynamically swinging pendulum rubber rod, subdivided into $N=10$ segments, circular cross section with radius $r=5.0 \mathrm{E}^{-3} \mathrm{~m}$ and end centroid $x_{0}(t) \equiv x_{0}$ fixed under gravity load with $g=9.81 \mathrm{~m} \mathrm{~s} s^{-2}$. The scenario is depicted in Figure 3. The rubber material parameters are chosen as $E=$ $5.0 \mathrm{E}^{+6} \mathrm{~N} \mathrm{~m}^{-2}, \nu=0.5$ and $\varrho=1.10 \mathrm{E}^{+3} \mathrm{~kg} \mathrm{~m}^{-3}$. All the examples in this section have been performed with shear correction factors equal to $\kappa_{1}=\kappa_{2}=1$.

Figure 4 shows excellent agreement compared to the corresponding ABAQUS 1D solution, computed with B31 Timoshenko shear flexible beam elements. It is seen that not only the positions/displacements, but as well the forces and moments are reflected accurately. The same applies to the frame positions, the (angular) velocities and the (angular) accelerations, which are not plotted here. Small model differences occur, since in ABAQUS shearing and extension are decoupled, which is not true for the Cosserat model.

Test 2 vs. ABAQUS (quasistatic) In a second test, we compare the quality of our discretisation scheme with the full 3D ABAQUS solution. To that end, a 3D continuum FE model of the rubber beam of Test 1 has been set up in ABAQUS. It is discretised with 160 (in longitudinal direction) $\times 12$ (in the cross section) continuum elements of type C3D8. (In total, these are 1920 elements.) We consider the scenario that is plotted in Figure 5. The scenario includes nontrivial coupling of bending and torsion. The fully clamped boundary conditions $\left(x_{0}(t), p_{0}(t)\right)$ and $\left(x_{N}(t), p_{N}(t)\right)$ are chosen in the way that the rod traverses the shape of the Greek letter $\gamma$ (front view) or the Greek letter $\Omega$ (side view). Figure 6 shows excellent agreement of our model results. We emphasise that for our discrete Cosserat rod model, we took only $N=10$ segments.

Performance Now we introduce some damping into the model, slight damping for bending and torsion, $c_{1}^{\dot{K}}=c_{2}^{\dot{K}}=2.0 \mathrm{E}^{-4} \mathrm{~kg} \mathrm{~m}^{3}, c_{3}^{\dot{K}}=8.0 \mathrm{E}^{-6} \mathrm{~kg} \mathrm{~m}^{3}$, and strong damping for shearing and extension $c_{1}^{\dot{\Gamma}}=c_{2}^{\dot{\Gamma}}=1.0 \mathrm{E}^{-1} \mathrm{~kg} \mathrm{~m}, c_{3}^{\dot{\Gamma}}=2.0 \mathrm{E}^{+2} \mathrm{~kg} \mathrm{~m}$. As we apply the multistep solver DASPK, we prefer the Baumgarte stabilisation technique to the projection technique. For the Baumgarte parameters, we choose $\omega=r=1.0 \mathrm{E}^{2} \mathrm{~s}^{-1}$, thus the constraint defect is critically damped without introducing significant additional stiffness into the model. 


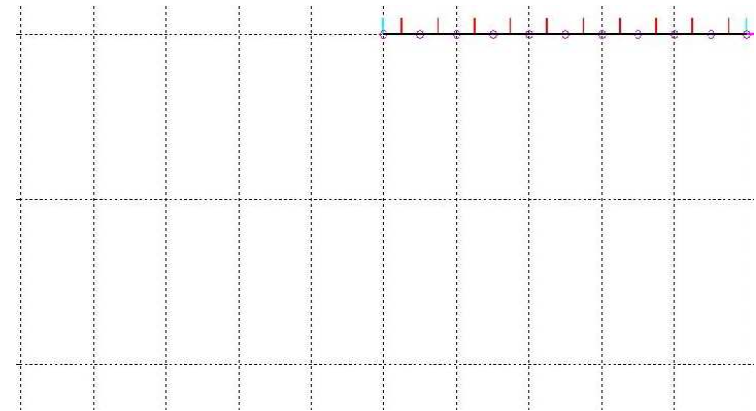

(a)

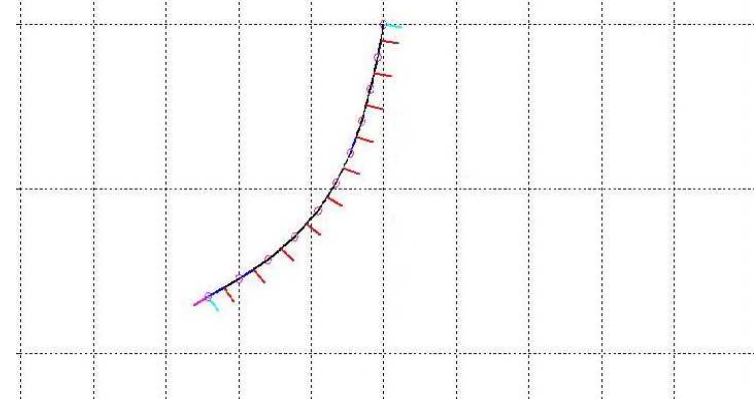

(c)

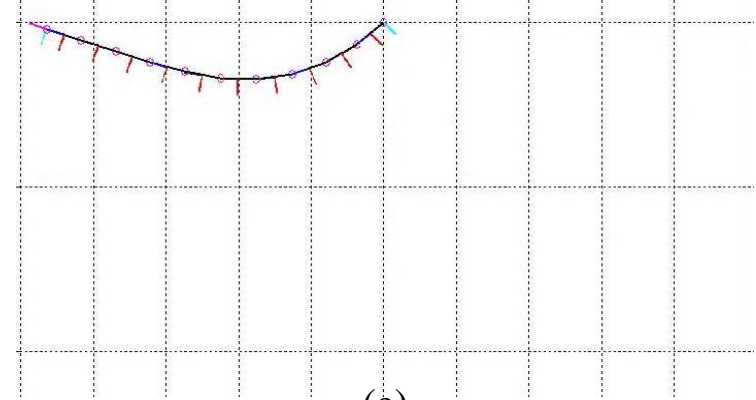

(e)

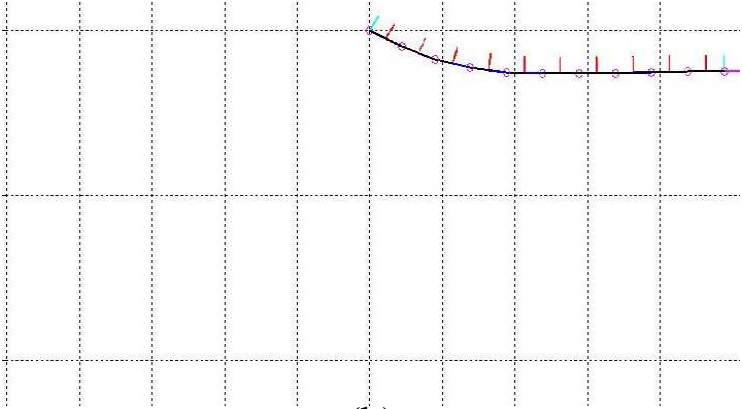

(b)

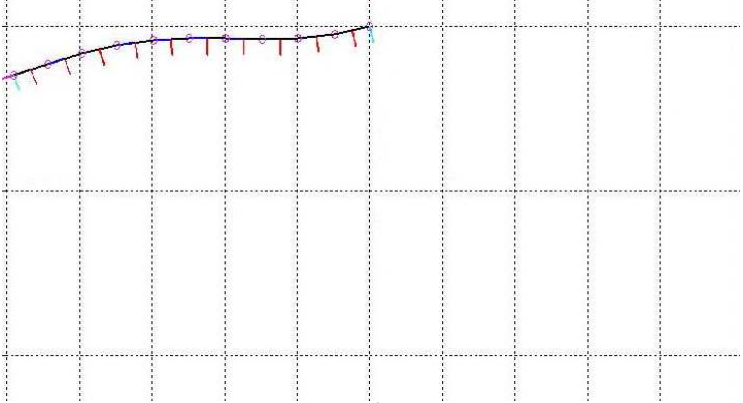

(d)

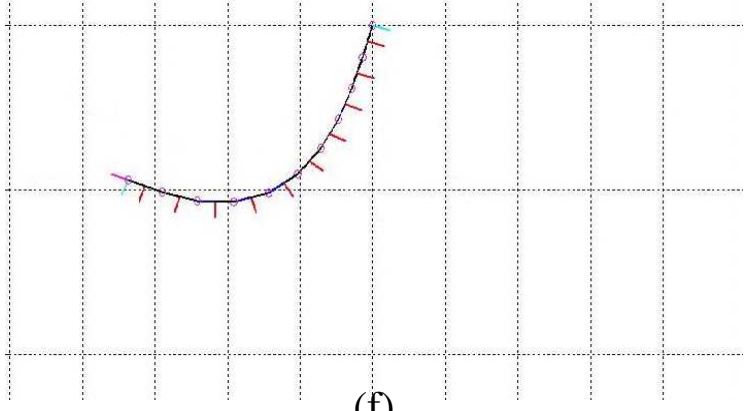

(f)

Figure 3: Snapshots of the dynamic Test 1.

For a rubber example, we choose the same swinging pendulum with fixed end centroid $x_{0}(t) \equiv x_{0}$ as in the comparative Test 1 vs. ABAQUS above. For a steel string example, we choose a string of length $L=1.0 \mathrm{~m}$ and radius $1.0 \mathrm{E}^{-3} \mathrm{~m}$ without precurvature, clamp in fully at $s=0$, i. e. $x_{0}(t) \equiv x_{0}$ and $p_{0}(t) \equiv p_{0}$, and subject it to gravity load. The material parameters for steel are set to $E=2.1 \mathrm{E}^{+6} \mathrm{Nm}^{-2}, \nu=0.2, \varrho=7.85 \mathrm{E}^{+3} \mathrm{~kg} \mathrm{~m}^{-3}$. In both examples, the number of unknowns in $q$ is equal to 70. Figure 7 shows the computational times for the solvers RADAU5 (an implicit Runge-Kutta method), SEUlEX (an extrapolation method), RoDAs (a Rosenbrock method), DoPRI5 (an explicit Runge-Kutta method) from [21, 22, 23, 34] and DASPK (= DASSL, a multistep BDF method) from [35] at several tolerances. For all the computations we choose TOL $=$ ABSTOL $=$ RELTOL, discarding the error control for the Lagrange multipliers. Clearly, the problem is stiff even for rubber material because of the presence of the stiff extensional and shearing springs. Thus, DOPRI5 fails, the corresponding step sizes indicate that it runs at the stability limit. In contrast to that, the four stiff solvers reveal satisfactory step size behaviour. For RADAU5, we discovered no significant difference between the classical and the Gustafsson step size strategy. For the solvers RADAu5, SEulEX and RodAs, we chose sparse linear algebra, adapted to second order ODEs, with upper resp. lower bandwidths MU- 

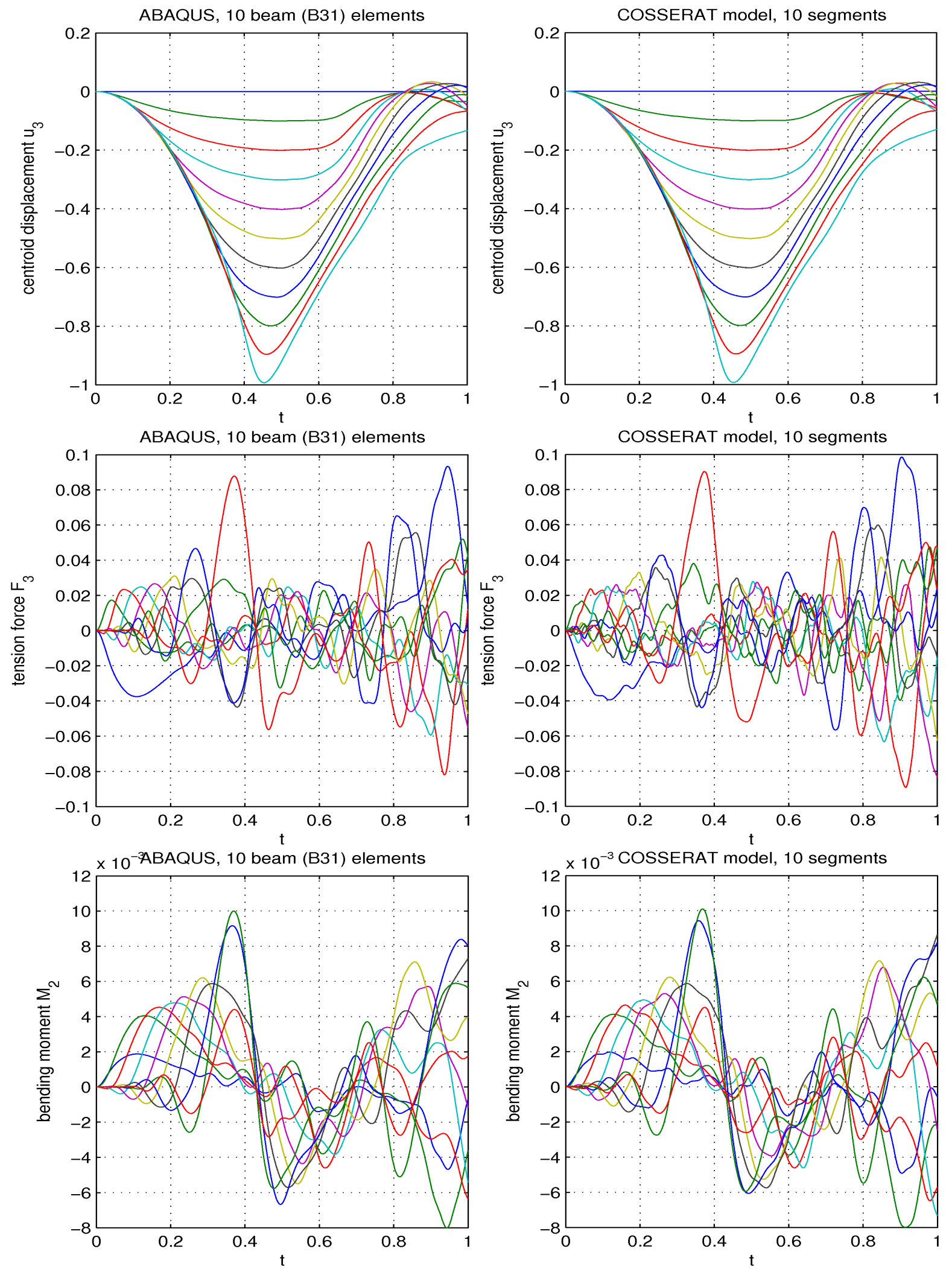

Figure 4: Dynamical comparison with ABAQUS 1D finite element solution (Test 1) 


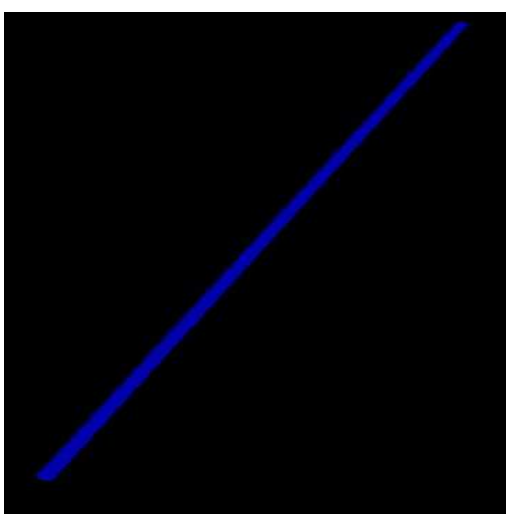

(a)

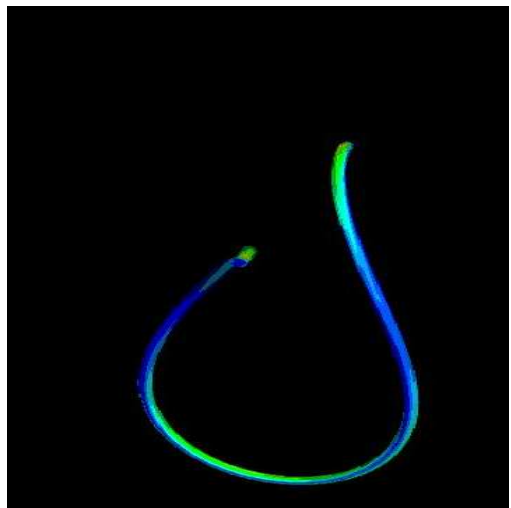

(d)

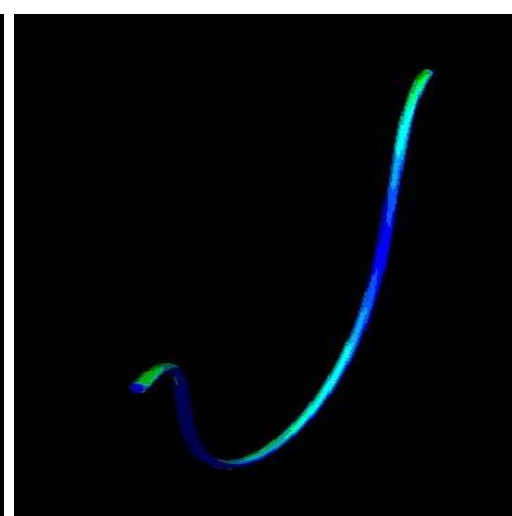

(b)

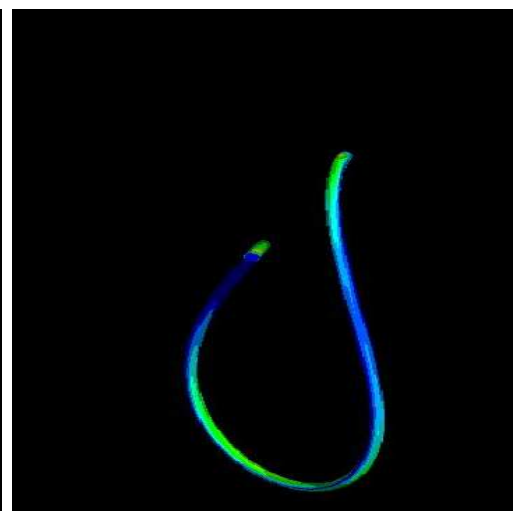

(e)

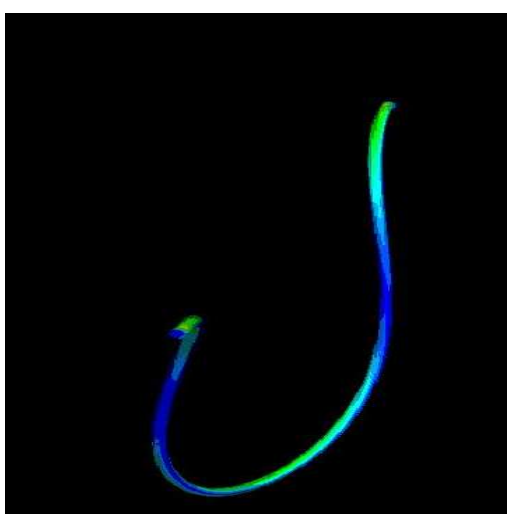

(c)

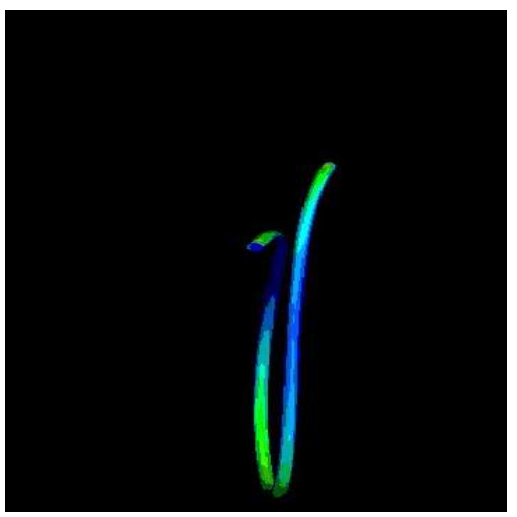

(f)

Figure 5: Snapshots of the quasistatic Test 2. (Colored is the Mises stress.)

JAC resp. MLJAC of the Jacobians $\partial f / \partial q$ and $\partial f / \partial v$ equal to ten. In both examples, we used a very robust discretisation of the curvature terms, motivated by the work [8]. RADAU5 spent $38 \%$ of the total computational time in order to evaluate $f$ and $\partial f / \partial u$, SEULEX 43\%, RoDAS $61 \%$ and DOPRI5 90\%; the remaining percentage is needed for (non)linear algebra. Roughly, an evaluation of $f$ needs about $1.06 \mathrm{E}^{-5} \mathrm{~s}$, which is comparable to [47], but with a much more robust curvature model, an evaluation of $\partial f / \partial u$ needs about $1.02 \mathrm{E}^{-4} \mathrm{~s}$, this is about ten times larger. Clearly, for coarse discretisations and rough error control during time integration, RoDAS performs best. Here, for the rubber pendulum example, the factor to the real physical time is 47 , for the steel string example 17 . For more stringent tolerances, RADAU5 performs best, since it is a high order method.

The computations have been performed on a $2.19 \mathrm{GHz}$ Dual Core AMD (Opteron) machine.

\section{CONCLUSIONS}

We presented an alternative discretisation approach for Cosserat rods that allows both very fast computations within milliseconds and accurate results compared to detailed finite element solutions. Our ansatz was based on quaternion parametrisation of rotations and a finite difference scheme on a staggered grid. The semi-discrete equations of motion were obtained as the Euler-Lagrange equations from a discrete variational principle. They yielded a consistent semidiscretisation to the continuous dynamical Cosserat partial differential equations of motion. For their numerical solution, we applied standard ODE / DAE solvers. 

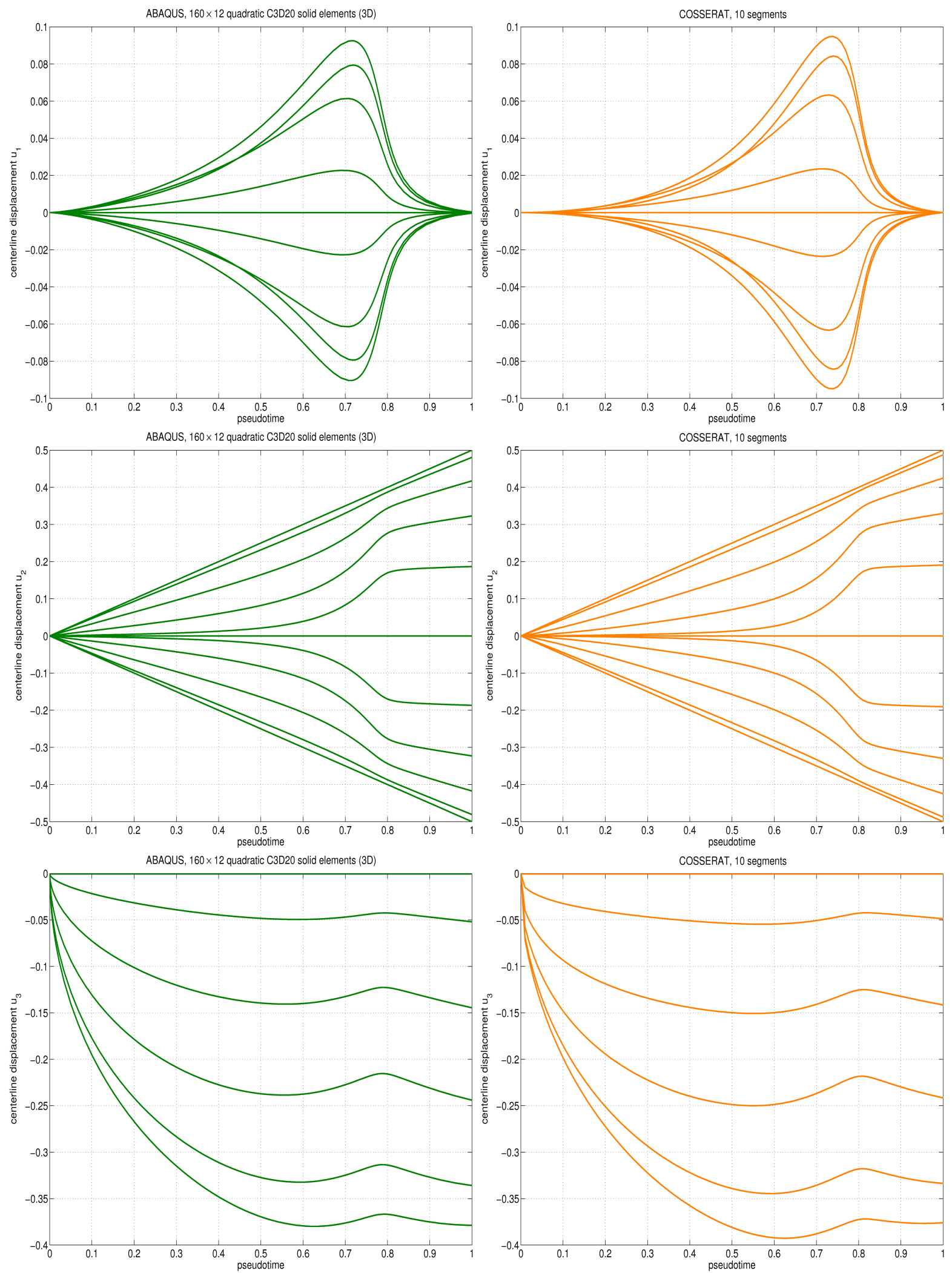

Figure 6: Quasistatic comparison with ABAQUS 3D finite element solution (Test 2) 

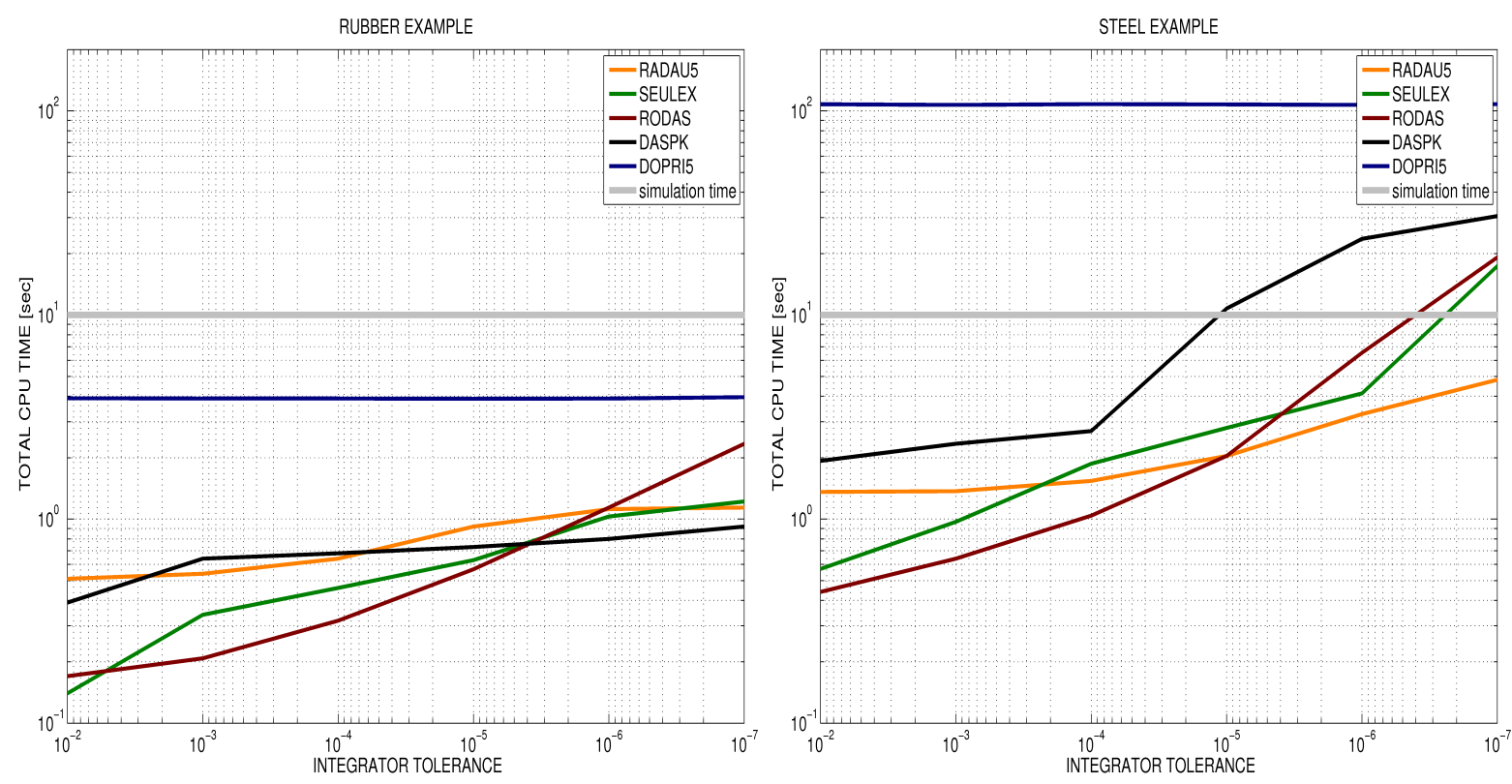

Figure 7: Computational times for different solvers.

\section{REFERENCES}

[1] Antman S. S.: The theory of rods. Springer, 1972.

[2] Antman S. S.: Dynamical problems for geometrically exact theories of nonlinearly viscoelastic rods. Journal of nonlinear science, 6, 1-18, 1996.

[3] Antman S.S.: Nonlinear problems of elasticity. Springer, 2005.

[4] Arnold M.: Numerical methods for simulation in applied mechanics. In: Simulation techniques for applied mechanics (Eds: Arnold M., Schiehlen W.), Springer, 191-246, 2008.

[5] Betsch P.: Computational methods for flexible multibody dynamics. Professorial dissertation, TU Kaiserslautern, 2002.

[6] Betsch P., Steinmann P.: A DAE approach to flexible multibody dynamics. Multibody system dynamics, 8, 365-389, 2002.

[7] Betsch P., Steinmann P.: Frame-indifferent beam finite elements based upon the geometrically exact beam theory. International journal for numerical methods in engineering, 54, 1755-1788, 2002.

[8] Bobenko A. I., Suris Y. B.: Discrete time Lagrangian mechanics on Lie groups with an application to the Lagrange top. Communications in mathematical physics, 204, 147-188, 1999.

[9] Cardona A., Géradin M.: A beam finite element nonlinear theory with finite rotations. International journal for numerical methods in engineering, 26, 2403-2434, 1998.

[10] Cardona A., Géradin M.: Flexible multibody dynamics. A finite element approach. Wiley, 2001.

[11] Cowper G. R.: The shear coefficient in Timoshenko's beam theory. Journal of applied mechanics, 68 (1), 87-92, 1968. 
[12] Craig R. R. jr., Kurdila A. J.: Fundamentals of structural mechanics. Wiley, 2006.

[13] Craig R. R.jr., Bampton M.C.C.: Coupling of substructures for dynamic analysis. AIAA journal, 6 (7), 1968.

[14] Crisfield M.A., Jelenic G.: Objectivity of strain measures in the geometrically exact threedimensional beam theory and its finite element implementation. Proceedings of the Royal Society London, 455, 1125-1147, 1999.

[15] Crisfield M. A.: Non-linear finite element analysis of solids and structures. Wiley, London, 1991.

[16] Dichmann D. J., Maddocks J.H.: An impetus-striction simulation of the dynamics of an elastica. Journal of nonlinear science, 6, 271-292, 1996.

[17] Ebbinghaus H.D. et al.: Numbers. Springer, 1992.

[18] Eich-Soellner E., Führer C.: Numerical methods in multibody dynamics. Teubner, 1998.

[19] Hanson A. J.: Visualizing quaternions. Elsevier, 2005.

[20] Hodges D. H.: Nonlinear composite beam theory. Progress in astronautics and aeronautics, 213, 2006.

[21] Hairer E., Lubich C., Roche M.: The numerical solutions of differential-algebraic systems by Runge-Kutta methods. Springer lecture notes in mathematics, 1409, 1989.

[22] Hairer E., Noersett S. P., Wanner G.: Solving ordinary differential equations I. Springer, 1993.

[23] Hairer E., Wanner G.: Solving ordinary differential equations II. Springer, 1996.

[24] Hairer E., Lubich C., Wanner G.: Geometric numerical integration. Springer, 2002.

[25] Ibrahimbegović A.: On finite element implementations of geometrically nonlinear Reissner's beam theory: Three dimensional curved beam elements. Computer methods in applied mechanics and engineering, 112, 11-26, 1995.

[26] Ibrahimbegović A., Frey F.: Finite element analysis of linear and nonlinear planar deformations of elastic initially curved beams. International journal for numerical methods in engineering, 36, 3239-3258, 1992.

[27] Kuipers J. B.: Quaternions and rotation sequences. Princeton university press, 1999.

[28] Kehrbaum S., Maddocks J.H.: Elastic rods, rigid bodies, quaternions and the last quadrature. Philosophical transactions of the royal society London A, 355, 2117-2136, 1997.

[29] Linn J., Stephan T., Carlsson J., Bohlin R.: Fast simulation of quasistatic rod deformations for VR applications. Report of the Fraunhofer ITWM, 143, 2008.

[30] Linn J., Stephan T.: Simulation of quasistatic deformations using discrete rod models. MULTIBODY DYNAMICS 2007, ECCOMAS Thematic Conference. Eds: C. L. Bottasso, P. Masarati, L. Trainelli, Milano, Italy, 25-28 June 2007.

[31] Lorenz M.: Optimization approaches for nonlinear rods. Diploma thesis, TU Kaiserslautern, 2008.

[32] Love A.E.H.: Treatise on the mathematical theory of elasticity. Dover, 1944. 
[33] Richtmyer R. D., Morton K. W.: Difference methods for initial value problems. Interscience publishers, New York, 1967.

[34] Lubich C.: Integration of stiff mechanical systems by Runge-Kutta methods. Journal of applied mathematics and physics, 44, 1022-1053, 1993.

[35] Petzold L. R.: A description of DASSL: A differential algebraic system solver. In: Scientific computing, Stepleman R. S. (Ed.), North-Holland, Amsterdam, 1981.

[36] Rabier P. J., Rheinboldt W. C.: Non-holonomic motion of rigid mechanical systems from a DAE viewpoint. SIAM, 2000.

[37] Roble D., Chan T.: Math in the entertainment industry. Mathematics unlimited, Engquist B. and Schmidt W. (Eds.), 971-990, 2001.

[38] Romero I.: The interpolation of rotations and its application to finite-element models of geometrically exact rods. Computational mechanics, 34, 121-133, 2004.

[39] Schiehlen W. O.: Multibody system dynamics: Roots and perspectives. Multibody system dynamics, 1, 149-188, 1997.

[40] Schiehlen W., Eberhard P.: Technische Dynamik. Modelle für Regelung und Simulation. Teubner, 2004.

[41] Schwab A. L., Meijaard P. J.: How to draw Euler angles and utilize Euler parameters. Proceedings of IDETC/CIE, 2008.

[42] Shabana A. A.: Flexible multibody dynamics: Review of past and recent developments. Multibody system dynamics, 1, 189-222, 1997.

[43] Shabana A. A.: Dynamics of multibody systems. Cambridge, 2005.

[44] Simeon B.: Numerical analysis of flexible multibody dynamics. Multibody system dynamics, 6 , 305-325, 2001.

[45] Simo J. C.: A finite strain beam formulation. The three dimensional dynamic problem. Part I. Computer methods in applied mechanics and engineering, 49, 55-70, 1985.

[46] Simo J. C., Vu-Quoc L.: A three dimensional finite strain rod model. Part II. Computer methods in applied mechanics and engineering, 58, 79-116, 1986.

[47] Spillmann J., Teschner M.: CoRdE. Cosserat rod elements for the dynamic simulation of onedimensional elastic objects. Eurographics/ACM SIGGRAPH, 1-10, 2007.

[48] Terzopoulos D., Platt J., Barr A., Zeltzer D., Witkin A., Blinn J.: Physically based modeling: Past, present and future. ACM SIGGRAPH, 23, 191-209, 1989. 


\section{Published reports of the Fraunhofer ITWM}

The PDF-files of the following reports are available under:

\section{www.itwm.fraunhofer.de/de/ zentral_berichte/berichte}

1. D. Hietel, K. Steiner, J. Struckmeier A Finite - Volume Particle Method for Compressible Flows

(19 pages, 1998)

2. M. Feldmann, S. Seibold

Damage Diagnosis of Rotors: Application of Hilbert Transform and Multi-Hypothesis Testing

Keywords: Hilbert transform, damage diagnosis,

Kalman filtering, non-linear dynamics

(23 pages, 1998)

3. Y. Ben-Haim, S. Seibold

Robust Reliability of Diagnostic Multi-

Hypothesis Algorithms: Application to

Rotating Machinery

Keywords: Robust reliability, convex models, Kalman filtering, multi-hypothesis diagnosis, rotating machinery, crack diagnosis

(24 pages, 1998)

4. F.-Th. Lentes, N. Siedow

Three-dimensional Radiative Heat Transfer in Glass Cooling Processes

(23 pages, 1998)

5. A. Klar, R. Wegener

A hierarchy of models for multilane vehicular traffic

Part I: Modeling

(23 pages, 1998)

Part II: Numerical and stochastic investigations (17 pages, 1998)

\section{A. Klar, N. Siedow}

Boundary Layers and Domain Decomposition for Radiative Heat Transfer and Diffusion Equations: Applications to Glass Manufacturing Processes

(24 pages, 1998

7. I. Choquet

Heterogeneous catalysis modelling and numerical simulation in rarified gas flows Part I: Coverage locally at equilibrium (24 pages, 1998)

8. J. Ohser, B. Steinbach, C. Lang

Efficient Texture Analysis of Binary Images

(17 pages, 1998)

9. J. Orlik

Homogenization for viscoelasticity of the integral type with aging and shrinkage (20 pages, 1998)

\section{J. Mohring}

Helmholtz Resonators with Large Aperture

(21 pages, 1998)

11. H. W. Hamacher, A. Schöbel

On Center Cycles in Grid Graphs

(15 pages, 1998)
12. H. W. Hamacher, K.-H. Küfer Inverse radiation therapy planning a multiple objective optimisation approach (14 pages, 1999)

13. C. Lang, J. Ohser, R. Hilfer

On the Analysis of Spatial Binary Images

(20 pages, 1999)

14. M. Junk

On the Construction of Discrete Equilibrium Distributions for Kinetic Schemes

(24 pages, 1999)

15. M. Junk, S. V. Raghurame Rao

A new discrete velocity method for NavierStokes equations

(20 pages, 1999)

16. H. Neunzert

Mathematics as a Key to Key Technologies

(39 pages (4 PDF-Files), 1999)

17. J. Ohser, K. Sandau

Considerations about the Estimation of the Size Distribution in Wicksell's Corpuscle Problem

(18 pages, 1999)

18. E. Carrizosa, H. W. Hamacher, R. Klein, S. Nickel

Solving nonconvex planar location problems by finite dominating sets Keywords: Continuous Location, Polyhedral Gauges, Finite Dominating Sets, Approximation, Sandwich Algorithm, Greedy Algorithm

(19 pages, 2000)

19. A. Becker

A Review on Image Distortion Measures Keywords: Distortion measure, human visual system (26 pages, 2000)

20. H. W. Hamacher, M. Labbé, S. Nickel T. Sonneborn

Polyhedral Properties of the Uncapacitated Multiple Allocation Hub Location Problem Keywords: integer programming, hub location, facility location, valid inequalities, facets, branch and cut (21 pages, 2000)

21. H. W. Hamacher, A. Schöbel

Design of Zone Tariff Systems in Public Transportation

(30 pages, 2001)

22. D. Hietel, M. Junk, R. Keck, D. Teleaga The Finite-Volume-Particle Method for Conservation Laws

(16 pages, 2001)

23. T. Bender, H. Hennes, J. Kalcsics, M. T. Melo, S. Nickel

Location Software and Interface with GIS and Supply Chain Management

Keywords: facility location, software development geographical information systems, supply chain management

(48 pages, 2001)

24. H. W. Hamacher, S. A. Tjandra

Mathematical Modelling of Evacuation

Problems: A State of Art

(44 pages, 2001)
25. J. Kuhnert, S. Tiwari

Grid free method for solving the Poisson equation

Keywords: Poisson equation, Least squares method, Grid free method

(19 pages, 2001)

26. T. Götz, H. Rave, D. Reinel-Bitzer,

K. Steiner, H. Tiemeier

Simulation of the fiber spinning process Keywords: Melt spinning, fiber model, Lattice Boltzmann, CFD

(19 pages, 2001)

\section{A. Zemitis}

On interaction of a liquid film with an obstacle Keywords: impinging jets, liquid film, models, numerical solution, shape

(22 pages, 2001)

\section{I. Ginzburg, K. Steiner}

Free surface lattice-Boltzmann method to model the filling of expanding cavities by Bingham Fluids

Keywords: Generalized LBE, free-surface phenomena, interface boundary conditions, filling processes, Bing ham viscoplastic model, regularized models

(22 pages, 2001)

\section{H. Neunzert}

"Denn nichts ist für den Menschen als Menschen etwas wert, was er nicht mit Leidenschaft tun kann"

Vortrag anlässlich der Verleihung des

Akademiepreises des Landes Rheinland-

Pfalz am 21.11.2001

Keywords: Lehre, Forschung, angewandte Mathematik

Mehrskalenanalyse, Strömungsmechanik

(18 pages, 2001)

30. J. Kuhnert, S. Tiwari

Finite pointset method based on the projection method for simulations of the incompressible Navier-Stokes equations

Keywords: Incompressible Navier-Stokes equations, Meshfree method, Projection method, Particle scheme, Least squares approximation

AMS subject classification: 76D05, 76M28

(25 pages, 2001)

31. R. Korn, M. Krekel

Optimal Portfolios with Fixed Consumption or Income Streams

Keywords: Portfolio optimisation, stochastic control

HJB equation, discretisation of control problems

(23 pages, 2002)

\section{M. Krekel}

Optimal portfolios with a loan dependent credit spread

Keywords: Portfolio optimisation, stochastic control, HJB equation, credit spread, log utility, power utility, non-linear wealth dynamic

(25 pages, 2002)

\section{J. Ohser, W. Nagel, K. Schladitz}

The Euler number of discretized sets - on the choice of adjacency in homogeneous lattices Keywords: image analysis, Euler number, neighborhod relationships, cuboidal lattice

32 pages, 2002

34. I. Ginzburg, K. Steiner

Lattice Boltzmann Model for Free-Surface flow and Its Application to Filling Process in Casting 
Keywords: Lattice Boltzmann models; free-surface phenomena; interface boundary conditions; filling processes; injection molding; volume of fluid method; interface boundary conditions; advection-schemes; upwind-schemes

(54 pages, 2002)

35. M. Günther, A. Klar, T. Materne, R. Wegen-

Multivalued fundamental diagrams and stop and go waves for continuum traffic equations Keywords: traffic flow, macroscopic equations, kinetic derivation, multivalued fundamental diagram, stop and go waves, phase transitions

(25 pages, 2002)

36. S. Feldmann, P. Lang, D. Prätzel-Wolters Parameter influence on the zeros of network determinants

Keywords: Networks, Equicofactor matrix polynomials, Realization theory, Matrix perturbation theory

(30 pages, 2002)

37. K. Koch, J. Ohser, K. Schladitz Spectral theory for random closed sets and estimating the covariance via frequency space

Keywords: Random set, Bartlett spectrum, fast Fourier transform, power spectrum

(28 pages, 2002)

38. D. d'Humières, I. Ginzburg

Multi-reflection boundary conditions for lattice Boltzmann models

Keywords: lattice Boltzmann equation, boudary condistions, bounce-back rule, Navier-Stokes equation

(72 pages, 2002)

\section{R. Korn}

Elementare Finanzmathematik

Keywords: Finanzmathematik, Aktien, Optionen, Portfolio-Optimierung, Börse, Lehrerweiterbildung, Mathematikunterricht

(98 pages, 2002)

40. J. Kallrath, M. C. Müller, S. Nickel

Batch Presorting Problems:

Models and Complexity Results

Keywords: Complexity theory, Integer programming,

Assigment, Logistics

(19 pages, 2002)

41. J. Linn

On the frame-invariant description of the phase space of the Folgar-Tucker equation Key words: fiber orientation, Folgar-Tucker equation, in jection molding

(5 pages, 2003)

\section{T. Hanne, S. Nickel}

A Multi-Objective Evolutionary Algorithm for Scheduling and Inspection Planning in Software Development Projects

Key words: multiple objective programming, project management and scheduling, software development evolutionary algorithms, efficient set (29 pages, 2003)

43. T. Bortfeld, K.-H. Küfer, M. Monz,

A. Scherrer, C. Thieke, H. Trinkaus

Intensity-Modulated Radiotherapy - A Large Scale Multi-Criteria Programming Problem Keywords: multiple criteria optimization, representative systems of Pareto solutions, adaptive triangulation clustering and disaggregation techniques, visualization of Pareto solutions, medical physics, external beam radiotherapy planning, intensity modulated radiotherapy (31 pages, 2003)
44. T. Halfmann, T. Wichmann

Overview of Symbolic Methods in Industria Analog Circuit Design

Keywords: CAD, automated analog circuit design, symbolic analysis, computer algebra, behavioral modeling system simulation, circuit sizing, macro modeling, differential-algebraic equations, index

(17 pages, 2003)

45. S. E. Mikhailov, J. Orlik

Asymptotic Homogenisation in Strength and Fatigue Durability Analysis of Composites

Keywords: multiscale structures, asymptotic homogenization, strength, fatique, singularity, non-local conditions

(14 pages, 2003)

46. P. Domínguez-Marín, P. Hansen, N. Mladenovi'c , S. Nickel

Heuristic Procedures for Solving the Discrete Ordered Median Problem

Keywords: genetic algorithms, variable neighborhood search, discrete facility location

(31 pages, 2003)

47. N. Boland, P. Domínguez-Marín, S. Nickel J. Puerto

Exact Procedures for Solving the Discrete Ordered Median Problem

Keywords: discrete location, Integer programming

(41 pages, 2003)

48. S. Feldmann, P. Lang

Padé-like reduction of stable discrete linear systems preserving their stability

Keywords: Discrete linear systems, model reduction,

stability, Hankel matrix, Stein equation

(16 pages, 2003)

49. J. Kallrath, S. Nicke

A Polynomial Case of the Batch Presorting Problem

Keywords: batch presorting problem, online optimization competetive analysis, polynomial algorithms, logistics

(17 pages, 2003)

\section{T. Hanne, H. L. Trinkaus}

knowCube for MCDM -

Visual and Interactive Support for

Multicriteria Decision Making

Key words: Multicriteria decision making, knowledge

management, decision support systems, visual interfac-

es, interactive navigation, real-life applications.

(26 pages, 2003)

51. O. lliev, V. Laptev

On Numerical Simulation of Flow Through

Oil Filters

Keywords: oil filters, coupled flow in plain and porous media, Navier-Stokes, Brinkman, numerical simulation (8 pages, 2003)

52. W. Dörfler, O. Iliev, D. Stoyanov, D. Vassileva On a Multigrid Adaptive Refinement Solver for Saturated Non-Newtonian Flow in Porous Media

Keywords: Nonlinear multigrid, adaptive refinement, non-Newtonian flow in porous media

(17 pages, 2003)

\section{S. Kruse}

On the Pricing of Forward Starting Options under Stochastic Volatility

Keywords: Option pricing, forward starting options,

Heston model, stochastic volatility, cliquet options

(11 pages, 2003)
54. O. Iliev, D. Stoyanov

Multigrid - adaptive local refinement solver for incompressible flows

Keywords: Navier-Stokes equations, incompressible flow projection-type splitting, SIMPLE, multigrid methods, adaptive local refinement, lid-driven flow in a cavity

(37 pages, 2003)

\section{V. Starikovicius}

The multiphase flow and heat transfer in porous media

Keywords: Two-phase flow in porous media, various formulations, global pressure, multiphase mixture model, numerical simulation

(30 pages, 2003)

56. P. Lang, A. Sarishvili, A. Wirsen

Blocked neural networks for knowledge extraction in the software development process Keywords: Blocked Neural Networks, Nonlinear Regression, Knowledge Extraction, Code Inspection

(21 pages, 2003)

57. H. Knaf, P. Lang, S. Zeiser

Diagnosis aiding in Regulation

Thermography using Fuzzy Logic

Keywords: fuzzy logic, knowledge representation,

expert system

(22 pages, 2003)

58. M. T. Melo, S. Nickel, F. Saldanha da Gama Largescale models for dynamic multicommodity capacitated facility location Keywords: supply chain management, strategic planning, dynamic location, modeling

(40 pages, 2003)

59. J. Orlik

Homogenization for contact problems with periodically rough surfaces

Keywords: asymptotic homogenization, contact problems (28 pages, 2004)

60. A. Scherrer, K.-H. Küfer, M. Monz, F. Alonso, T. Bortfeld

IMRT planning on adaptive volume structures - a significant advance of computational complexity

Keywords: Intensity-modulated radiation therapy (IMRT), inverse treatment planning, adaptive volume structures, hierarchical clustering, local refinement, adaptive clustering, convex programming, mesh generation, multi-grid methods

(24 pages, 2004)

61. D. Kehrwald

Parallel lattice Boltzmann simulation of complex flows

Keywords: Lattice Boltzmann methods, parallel computing, microstructure simulation, virtual material design, pseudo-plastic fluids, liquid composite moulding (12 pages, 2004)

62. O. Iliev, J. Linn, M. Moog, D. Niedziela, V. Starikovicius

On the Performance of Certain Iterative Solvers for Coupled Systems Arising in Discretization of Non-Newtonian Flow Equations

Keywords: Performance of iterative solvers, Preconditioners, Non-Newtonian flow

(17 pages, 2004)

63. R. Ciegis, O. Iliev, S. Rief, K. Steiner On Modelling and Simulation of Different Regimes for Liquid Polymer Moulding 
Keywords: Liquid Polymer Moulding, Modelling, Simulation, Infiltration, Front Propagation, non-Newtonian flow in porous media

(43 pages, 2004)

64. T. Hanne, H. Neu

Simulating Human Resources in

Software Development Processes

Keywords: Human resource modeling, software process, productivity, human factors, learning curve

(14 pages, 2004)

65. O. Iliev, A. Mikelic, P. Popov

Fluid structure interaction problems in deformable porous media: Toward permeability of deformable porous media

Keywords: fluid-structure interaction, deformable porous media, upscaling, linear elasticity, stokes, finite elements

(28 pages, 2004)

66. F. Gaspar, O. Iliev, F. Lisbona, A. Naumovich, P. Vabishchevich

On numerical solution of 1-D poroelasticity equations in a multilayered domain

Keywords: poroelasticity, multilayered material, finite

volume discretization, MAC type grid

(41 pages, 2004)

67. J. Ohser, K. Schladitz, K. Koch, M. Nöthe Diffraction by image processing and its application in materials science

Keywords: porous microstructure, image analysis, random set, fast Fourier transform, power spectrum, Bartlett spectrum

(13 pages, 2004)

68. H. Neunzert

Mathematics as a Technology: Challenges for the next 10 Years

Keywords: applied mathematics, technology, modelling, simulation, visualization, optimization, glass processing, spinning processes, fiber-fluid interaction, trubulence effects, topological optimization, multicriteria optimization, Uncertainty and Risk, financial mathematics, Malliavin calculus, Monte-Carlo methods, virtual material design, filtration, bio-informatics, system biology (29 pages, 2004)

69. R. Ewing, O. lliev, R. Lazarov, A. Naumovich On convergence of certain finite difference discretizations for 1D poroelasticity interface problems

Keywords: poroelasticity, multilayered material, finite volume discretizations, MAC type grid, error estimates (26 pages,2004)

70. W. Dörfler, O. Iliev, D. Stoyanov, D. Vassileva On Efficient Simulation of Non-Newtonian Flow in Saturated Porous Media with a Multigrid Adaptive Refinement Solver Keywords: Nonlinear multigrid, adaptive renement, non-Newtonian in porous media

(25 pages, 2004)

71. J. Kalcsics, S. Nickel, M. Schröder Towards a Unified Territory Design Approach - Applications, Algorithms and GIS Integration Keywords: territory desgin, political districting, sales territory alignment, optimization algorithms, Geographical Information Systems

(40 pages, 2005)

72. K. Schladitz, S. Peters, D. Reinel-Bitzer

A. Wiegmann, J. Ohser

Design of acoustic trim based on geometric modeling and flow simulation for non-woven
Keywords: random system of fibers, Poisson line process, flow resistivity, acoustic absorption, Lattice-Boltzmann method, non-woven (21 pages, 2005)

73. V. Rutka, A. Wiegmann

Explicit Jump Immersed Interface Method for virtual material design of the effective elastic moduli of composite materials Keywords: virtual material design, explicit jump immersed interface method, effective elastic moduli composite materials

(22 pages, 2005)

\section{T. Hanne}

Eine Übersicht zum Scheduling von Baustellen Keywords: Projektplanung, Scheduling, Bauplanung, Bauindustrie

(32 pages, 2005)

\section{J. Linn}

The Folgar-Tucker Model as a Differetial Algebraic System for Fiber Orientation Calculation

Keywords: fiber orientation, Folgar-Tucker model, invariants, algebraic constraints, phase space, trace stability

(15 pages, 2005)

76. M. Speckert, K. Dreßler, H. Mauch, A. Lion, G. J. Wierda

Simulation eines neuartigen Prüfsystems für Achserprobungen durch MKS-Modellierung einschließlich Regelung Keywords: virtual test rig, suspension testing, multibody simulation, modeling hexapod test rig, optmization of test rig configuration (20 pages, 2005)

77. K.-H. Küfer, M. Monz, A. Scherrer, P. Süss, F. Alonso, A. S. A. Sultan, Th. Bortfeld,

D. Craft, Chr. Thieke

Multicriteria optimization in intensity modulated radiotherapy planning Keywords: multicriteria optimization, extreme solutions, real-time decision making, adaptive approximation schemes, clustering methods, IMRT planning, reverse engineering

(51 pages, 2005)

\section{S. Amstutz, H. Andrä}

A new algorithm for topology optimization using a level-set method

Keywords: shape optimization, topology optimization topological sensitivity, level-set

(22 pages, 2005)

\section{N. Ettrich}

Generation of surface elevation models for urban drainage simulation

Keywords: Flooding, simulation, urban elevation

models, laser scanning

(22 pages, 2005)

80. H. Andrä, J. Linn, I. Matei, I. Shklyar,

K. Steiner, E. Teichmann

OPTCAST - Entwicklung adäquater Struk turoptimierungsverfahren für Gießereien Technischer Bericht (KURZFASSUNG)

Keywords: Topologieoptimierung, Level-Set-Methode, Gießprozesssimulation, Gießtechnische Restriktionen,

CAE-Kette zur Strukturoptimierung

(77 pages, 2005)

81. N. Marheineke, R. Wegener Fiber Dynamics in Turbulent Flows

Part I: General Modeling Framework
Keywords: fiber-fluid interaction: Cosserat rod: turbulence modeling; Kolmogorov's energy spectrum; double-velocity correlations; differentiable Gaussian fields (20 pages, 2005)

\section{Part II: Specific Taylor Drag}

Keywords: flexible fibers; $k-\varepsilon$ turbulence model; fiber-turbulence interaction scales; air drag; random Gaussian aerodynamic force; white noise; stochastic differential equations; ARMA process

(18 pages, 2005)

\section{C. H. Lampert, O. Wirjadi}

An Optimal Non-Orthogonal Separation of the Anisotropic Gaussian Convolution Filter Keywords: Anisotropic Gaussian filter, linear filtering, ori entation space, $n D$ image processing, separable filters (25 pages, 2005)

\section{H. Andrä, D. Stoyanov}

Error indicators in the parallel finite element solver for linear elasticity DDFEM Keywords: linear elasticity, finite element method, hierarchical shape functions, domain decom-position, parallel implementation, a posteriori error estimates (21 pages, 2006)

84. M. Schröder, I. Solchenbach Optimization of Transfer Quality in Regional Public Transit

Keywords: public transit, transfer quality, quadratic assignment problem

(16 pages, 2006)

85. A. Naumovich, F. J. Gaspar

On a multigrid solver for the three-dimensional Biot poroelasticity system in multilayered domains

Keywords: poroelasticity, interface problem, multigrid, operator-dependent prolongation

(11 pages, 2006)

86. S. Panda, R. Wegener, N. Marheineke Slender Body Theory for the Dynamics of Curved Viscous Fibers

Keywords: curved viscous fibers; fluid dynamics; NavierStokes equations; free boundary value problem; asymptotic expansions; slender body theory

(14 pages, 2006)

87. E. Ivanov, H. Andrä, A. Kudryavtsev Domain Decomposition Approach for Automatic Parallel Generation of Tetrahedral Grids Key words: Grid Generation, Unstructured Grid, Delaunay Triangulation, Parallel Programming, Domain Decomposition, Load Balancing

(18 pages, 2006)

88. S. Tiwari, S. Antonov, D. Hietel, J. Kuhnert, R. Wegener

A Meshfree Method for Simulations of Interactions between Fluids and Flexible Structures

Key words: Meshfree Method, FPM, Fluid Structure Interaction, Sheet of Paper, Dynamical Coupling (16 pages, 2006)

89. R. Ciegis, O. Iliev, V. Starikovicius, K. Steiner Numerical Algorithms for Solving Problems of Multiphase Flows in Porous Media

Keywords: nonlinear algorithms, finite-volume method, software tools, porous media, flows

(16 pages, 2006)

90. D. Niedziela, O. Iliev, A. Latz

On 3D Numerical Simulations of Viscoelastic Fluids 
Keywords: non-Newtonian fluids, anisotropic viscosity integral constitutive equation

(18 pages, 2006)

\section{A. Winterfeld}

Application of general semi-infinite Programming to Lapidary Cutting Problems Keywords: large scale optimization, nonlinear programming, general semi-infinite optimization, design centering, clustering

(26 pages, 2006)

\section{J. Orlik, A. Ostrovska}

Space-Time Finite Element Approximation and Numerical Solution of Hereditary Linear Viscoelasticity Problems

Keywords: hereditary viscoelasticity; kern approximation by interpolation; space-time finite element approximation, stability and a priori estimate

(24 pages, 2006)

93. V. Rutka, A. Wiegmann, H. Andrä EJIIM for Calculation of effective Elastic Moduli in 3D Linear Elasticity

Keywords: Elliptic PDE, linear elasticity, irregular domain, finite differences, fast solvers, effective elastic moduli

(24 pages, 2006)

94. A. Wiegmann, A. Zemitis

EJ-HEAT: A Fast Explicit Jump Harmonic Averaging Solver for the Effective Heat Conductivity of Composite Materials Keywords: Stationary heat equation, effective thermal conductivity, explicit jump, discontinuous coefficients, virtual material design, microstructure simulation, EJ-HEAT

(21 pages, 2006)

\section{A. Naumovich}

On a finite volume discretization of the three-dimensional Biot poroelasticity system in multilayered domains

Keywords: Biot poroelasticity system, interface problems, finite volume discretization, finite difference method (21 pages, 2006)

96. M. Krekel, J. Wenzel

A unified approach to Credit Default Swaption and Constant Maturity Credit Default Swap valuation

Keywords: LIBOR market model, credit risk, Credit Default Swaption, Constant Maturity Credit Default Swapmethod

(43 pages, 2006)

\section{A. Dreyer}

\section{Interval Methods for Analog Circiuts}

Keywords: interval arithmetic, analog circuits, tolerance analysis, parametric linear systems, frequency response, symbolic analysis, CAD, computer algebra

(36 pages, 2006)

98. N. Weigel, S. Weihe, G. Bitsch, K. Dreßler Usage of Simulation for Design and Optimization of Testing

Keywords: Vehicle test rigs, MBS, control, hydraulics, testing philosophy

(14 pages, 2006)

99. H. Lang, G. Bitsch, K. Dreßler, M. Speckert Comparison of the solutions of the elastic and elastoplastic boundary value problems Keywords: Elastic BVP, elastoplastic BVP, variational inequalities, rate-independency, hysteresis, linear kinematic hardening, stop- and play-operator (21 pages, 2006)
100. M. Speckert, K. Dreßler, H. Mauch MBS Simulation of a hexapod based suspension test rig

Keywords: Test rig, MBS simulation, suspension,

hydraulics, controlling, design optimization

(12 pages, 2006)

101. S. Azizi Sultan, K.-H. Küfer

A dynamic algorithm for beam orientations in multicriteria IMRT planning

Keywords: radiotherapy planning, beam orientation optimization, dynamic approach, evolutionary algo-

rithm, global optimization

(14 pages, 2006)

102. T. Götz, A. Klar, N. Marheineke, R. Wegener A Stochastic Model for the Fiber Lay-down Process in the Nonwoven Production Keywords: fiber dynamics, stochastic Hamiltonian system, stochastic averaging

(17 pages, 2006)

103. Ph. Süss, K.-H. Küfer

Balancing control and simplicity: a variable aggregation method in intensity modulated radiation therapy planning

Keywords: IMRT planning, variable aggregation, clus-

tering methods

(22 pages, 2006)

104. A. Beaudry, G. Laporte, T. Melo, S. Nickel Dynamic transportation of patients in hospitals

Keywords: in-house hospital transportation, dial-a-ride, dynamic mode, tabu search

(37 pages, 2006)

\section{Th. Hanne}

Applying multiobjective evolutionary algorithms in industrial projects

Keywords: multiobjective evolutionary algorithms, discrete optimization, continuous optimization, electronic circuit design, semi-infinite programming, scheduling (18 pages, 2006)

106. J. Franke, S. Halim

Wild bootstrap tests for comparing signals and images

Keywords: wild bootstrap test, texture classification, textile quality control, defect detection, kernel estimate, nonparametric regression

(13 pages, 2007)

\section{Z. Drezner, S. Nickel}

Solving the ordered one-median problem in the plane

Keywords: planar location, global optimization, ordered median, big triangle small triangle method, bounds, numerical experiments

(21 pages, 2007)

108. Th. Götz, A. Klar, A. Unterreiter, R. Wegener

Numerical evidance for the non-existing of solutions of the equations desribing rotational fiber spinning

Keywords: rotational fiber spinning, viscous fibers, boundary value problem, existence of solutions (11 pages, 2007)

\section{Ph. Süss, K.-H. Küfer}

Smooth intensity maps and the BortfeldBoyer sequencer

Keywords: probabilistic analysis, intensity modulated radiotherapy treatment (IMRT), IMRT plan application, step-and-shoot sequencing

(8 pages, 2007)
110. E. Ivanov, O. Gluchshenko, H. Andrä, A. Kudryavtsev

Parallel software tool for decomposing and meshing of $3 d$ structures

Keywords: a-priori domain decomposition, unstructured grid, Delaunay mesh generation

(14 pages, 2007)

111. O. lliev, R. Lazarov, J. Willems Numerical study of two-grid preconditioners for 1d elliptic problems with highly oscillating discontinuous coefficients Keywords: two-grid algorithm, oscillating coefficients, preconditioner

(20 pages, 2007)

112. L. Bonilla, T. Götz, A. Klar, N. Marheineke, R. Wegener

Hydrodynamic limit of the Fokker-Planckequation describing fiber lay-down processes

Keywords: stochastic dierential equations, FokkerPlanck equation, asymptotic expansion, OrnsteinUhlenbeck process

(17 pages, 2007)

113. S. Rief

Modeling and simulation of the pressing section of a paper machine

Keywords: paper machine, computational fluid dynamics, porous media

(41 pages, 2007)

114. R. Ciegis, O. Iliev, Z. Lakdawala On parallel numerical algorithms for simulating industrial filtration problems Keywords: Navier-Stokes-Brinkmann equations, finite volume discretization method, SIMPLE, parallel computing, data decomposition method

(24 pages, 2007)

115. N. Marheineke, R. Wegener

Dynamics of curved viscous fibers with surface tension

Keywords: Slender body theory, curved viscous bers with surface tension, free boundary value problem (25 pages, 2007)

116. S. Feth, J. Franke, M. Speckert Resampling-Methoden zur mse-Korrektur und Anwendungen in der Betriebsfestigkeit Keywords: Weibull, Bootstrap, Maximum-Likelihood, Betriebsfestigkeit

(16 pages, 2007)

\section{H. Knaf}

Kernel Fisher discriminant functions - a concise and rigorous introduction

Keywords: wild bootstrap test, texture classification, textile quality control, defect detection, kernel estimate, nonparametric regression

(30 pages, 2007)

118. O. Iliev, I. Rybak

On numerical upscaling for flows in heterogeneous porous media

Keywords: numerical upscaling, heterogeneous porous media, single phase flow, Darcy's law, multiscale problem, effective permeability, multipoint flux approximation, anisotropy

(17 pages, 2007)

119. O. Iliev, I. Rybak

On approximation property of multipoint flux approximation method 
Keywords: Multipoint flux approximation, finite volume method, elliptic equation, discontinuous tensor coefficients, anisotropy

(15 pages, 2007)

120. O. Iliev, I. Rybak, J. Willems

On upscaling heat conductivity for a class of industrial problems

Keywords: Multiscale problems, effective heat conduc-

tivity, numerical upscaling, domain decomposition

(21 pages, 2007)

121. R. Ewing, O. Iliev, R. Lazarov, I. Rybak

On two-level preconditioners for flow in porous media

Keywords: Multiscale problem, Darcy's law, single phase flow, anisotropic heterogeneous porous media,

numerical upscaling, multigrid, domain decomposition, efficient preconditioner

(18 pages, 2007)

\section{M. Brickenstein, A. Dreyer}

POLYBORI: A Gröbner basis framework for Boolean polynomials

Keywords: Gröbner basis, formal verification, Boolean polynomials, algebraic cryptoanalysis, satisfiability

(23 pages, 2007)

\section{O. Wirjad}

Survey of 3d image segmentation methods Keywords: image processing, 3d, image segmentation, binarization

(20 pages, 2007)

\section{S. Zeytun, A. Gupta}

A Comparative Study of the Vasicek and the CIR Model of the Short Rate

Keywords: interest rates, Vasicek model, CIR-model, calibration, parameter estimation

(17 pages, 2007)

\section{G. Hanselmann, A. Sarishvili}

Heterogeneous redundancy in software quality prediction using a hybrid Bayesian approach

Keywords: reliability prediction, fault prediction, nonhomogeneous poisson process, Bayesian model averaging

(17 pages, 2007)

126. V. Maag, M. Berger, A. Winterfeld, K.-H. Küfer

A novel non-linear approach to minimal area rectangular packing

Keywords: rectangular packing, non-overlapping constraints, non-linear optimization, regularization, relaxation

(18 pages, 2007)

127. M. Monz, K.-H. Küfer, T. Bortfeld, C. Thieke Pareto navigation - systematic multi-criteria-based IMRT treatment plan determination

Keywords: convex, interactive multi-objective optimization, intensity modulated radiotherapy planning (15 pages, 2007)

128. M. Krause, A. Scherrer

On the role of modeling parameters in IMRT plan optimization

Keywords: intensity-modulated radiotherapy (IMRT), inverse IMRT planning, convex optimization, sensitivity analysis, elasticity, modeling parameters, equivalent uniform dose (EUD)

(18 pages, 2007)
129. A. Wiegmann

Computation of the permeability of porous materials from their microstructure by FFFStokes

Keywords: permeability, numerical homogenization fast Stokes solver

(24 pages, 2007)

130. T. Melo, S. Nickel, F. Saldanha da Gama Facility Location and Supply Chain Management - A comprehensive review

Keywords: facility location, supply chain management, network design

(54 pages, 2007)

131. T. Hanne, T. Melo, S. Nickel

Bringing robustness to patient flow management through optimized patient transports in hospitals

Keywords: Dial-a-Ride problem, online problem, case study, tabu search, hospital logistics

(23 pages, 2007)

132. R. Ewing, O. Iliev, R. Lazarov, I. Rybak, J. Willems

An efficient approach for upscaling properties of composite materials with high contrast of coefficients

Keywords: effective heat conductivity, permeability of fractured porous media, numerical upscaling, fibrous insulation materials, metal foams

(16 pages, 2008)

133. S. Gelareh, S. Nickel

New approaches to hub location problems in public transport planning

Keywords: integer programming, hub location, transportation, decomposition, heuristic

(25 pages, 2008)

134. G. Thömmes, J. Becker, M. Junk, A. K. Vaikuntam, D. Kehrwald, A. Klar, K. Steiner,

A. Wiegmann

A Lattice Boltzmann Method for immiscible multiphase flow simulations using the Level Set Method

Keywords: Lattice Boltzmann method, Level Set method free surface, multiphase flow

(28 pages, 2008)

\section{J. Orlik}

\section{Homogenization in elasto-plasticity}

Keywords: multiscale structures, asymptotic homogenization, nonlinear energy

(40 pages, 2008)

136. J. Almquist, H. Schmidt, P. Lang, J. Deitmer, M. Jirstrand, D. Prätzel-Wolters, H. Becker

Determination of interaction between

MCT1 and CAII via a mathematical and physiological approach

Keywords: mathematical modeling; model reduction; electrophysiology; $\mathrm{pH}$-sensitive microelectrodes; proton antenna

(20 pages, 2008)

137. E. Savenkov, H. Andrä, O. Iliev

An analysis of one regularization approach for solution of pure Neumann problem Keywords: pure Neumann problem, elasticity, regularization, finite element method, condition number (27 pages, 2008)

138. O. Berman, J. Kalcsics, D. Krass, S. Nicke The ordered gradual covering location problem on a network
Keywords: gradual covering, ordered median function, network location

(32 pages, 2008)

139. S. Gelareh, S. Nickel

Multi-period public transport design: A novel model and solution approaches Keywords: Integer programming, hub location, public transport, multi-period planning, heuristics (31 pages, 2008)

140. T. Melo, S. Nickel, F. Saldanha-da-Gama Network design decisions in supply chain planning

Keywords: supply chain design, integer programming models, location models, heuristics

(20 pages, 2008)

141. C. Lautensack, A. Särkkä, J. Freitag, K. Schladitz

Anisotropy analysis of pressed point processes

Keywords: estimation of compression, isotropy test nearest neighbour distance, orientation analysis, polar ice, Ripley's K function

(35 pages, 2008)

142. O. Iliev, R. Lazarov, J. Willems

A Graph-Laplacian approach for calculating the effective thermal conductivity of complicated fiber geometries

Keywords: graph laplacian, effective heat conductivity, numerical upscaling fibrous materials

(14 pages, 2008)

143. J. Linn, T. Stephan, J. Carlsson, R. Bohlin Fast simulation of quasistatic rod deformations for VR applications

Keywords: quasistatic deformations, geometrically exact rod models, variational formulation, energy min imization, finite differences, nonlinear conjugate gradients

(7 pages, 2008)

\section{J. Linn, T. Stephan}

Simulation of quasistatic deformations using discrete rod models

Keywords: quasistatic deformations, geometrically exact rod models, variational formulation, energy minimization, finite differences, nonlinear conjugate gradients

(9 pages, 2008)

145. J. Marburger, N. Marheineke, R. Pinnau Adjoint based optimal control using meshless discretizations

Keywords: Mesh-less methods, particle methods, Eulerian-Lagrangian formulation, optimization strategies, adjoint method, hyperbolic equations

(14 pages, 2008

146. S. Desmettre, J. Gould, A. Szimayer Own-company stockholding and work effort preferences of an unconstrained executive Keywords: optimal portfolio choice, executive compensation

(33 pages, 2008)

147. M. Berger, M. schröder, K.-H. Küfer

A constraint programming approach for the two-dimensional rectangular packing problem with orthogonal orientations

Keywords: rectangular packing, orthogonal orientations non-overlapping constraints, constraint propagation

(13 pages, 2008) 
148. K. Schladitz, C. Redenbach, T. Sych, M. Godehardt

Microstructural characterisation of open foams using $3 d$ images

Keywords: virtual material design, image analysis, open foams

(30 pages, 2008)

149. E. Fernández, J. Kalcsics, S. Nickel, R. Ríos-Mercado

A novel territory design model arising in the implementation of the WEEE-Directive Keywords: heuristics, optimization, logistics, recycling (28 pages, 2008)

150. H. Lang, J. Linn

Lagrangian field theory in space-time for geometrically exact Cosserat rods

Keywords: Cosserat rods, geometrically exact rods, small strain, large deformation, deformable bodies, Lagrangian field theory, variational calculus

(19 pages, 2009)

151. K. Dreßler, M. Speckert, R. Müller, Ch. Weber

Customer loads correlation in truck engineering

Keywords: Customer distribution, safety critical components, quantile estimation, Monte-Carlo methods (11 pages, 2009)

152. H. Lang, K. Dreßler

An improved multiaxial stress-strain correction model for elastic FE postprocessing

Keywords: Jiang's model of elastoplasticity, stress-strain correction, parameter identification, automatic differentiation, least-squares optimization, Coleman-Li algorithm

(6 pages, 2009)

153. J. Kalcsics, S. Nickel, M. Schröder A generic geometric approach to territory design and districting

Keywords: Territory design, districting, combinatorial optimization, heuristics, computational geometry

(32 pages, 2009)

154. Th. Fütterer, A. Klar, R. Wegener

An energy conserving numerical scheme for the dynamics of hyperelastic rods

Keywords: Cosserat rod, hyperealstic, energy conservation, finite differences

(16 pages, 2009)

155. A. Wiegmann, L. Cheng, E. Glatt, O. lliev, S. Rief

Design of pleated filters by computer simulations

Keywords: Solid-gas separation, solid-liquid separation, pleated filter, design, simulation

(21 pages, 2009)

156. A. Klar, N. Marheineke, R. Wegener

Hierarchy of mathematical models for production processes of technical textiles

Keywords: Fiber-fluid interaction, slender-body theory, turbulence modeling, model reduction, stochastic differential equations, Fokker-Planck equation, asymptotic expansions, parameter identification

(21 pages, 2009)

157. E. Glatt, S. Rief, A. Wiegmann, M. Knefel, E. Wegenke

Structure and pressure drop of real and virtual metal wire meshes
Keywords: metal wire mesh, structure simulation model calibration, CFD simulation, pressure loss (7 pages, 2009)

158. S. Kruse, M. Müller

Pricing American call options under the assumption of stochastic dividends - An application of the Korn-Rogers model

Keywords: option pricing, American options, dividends, dividend discount model, Black-Scholes model (22 pages, 2009)

159. H. Lang, J. Linn, M. Arnold

Multibody dynamics simulation of geometrically exact Cosserat rods

Keywords: flexible multibody dynamics, large deformations, finite rotations, constrained mechanical systems, structural dynamics

(20 pages, 2009)

Status quo: May 2009 